\title{
Three-dimensional dendritic needle network model for alloy solidification
}

\author{
D. Tourret* \\ Los Alamos National Laboratory, Materials Science and Technology Division, Los Alamos, NM 87545, USA \\ A. Karma* \\ Department of Physics and Center for Interdisciplinary Research on Complex Systems, \\ Northeastern University, Boston, MA 02115, USA
}

\begin{abstract}
We present a three-dimensional (3D) formulation of the multiscale Dendritic Needle Network (DNN) model for dendritic microstructure growth. This approach is aimed at simulating quantitatively the solidification dynamics of complex hierarchical networks in spatially extended dendritic arrays, hence bridging the scale gap between phase-field simulations at the scale of a few dendrites and coarse-grained simulations on the larger scale of entire polycrystalline structures. In the DNN model, the dendritic network is represented by a network of branches that interact through the solutal diffusion field. The tip velocity $V(t)$ and tip radius $\rho(t)$ of each needle is determined by combining a standard solvability condition that fixes the product $\rho^{2} V$ and a solute flux conservation condition that fixes the product $\rho V^{2}$ in $2 \mathrm{D}$ and $\rho V$ in 3D as a function of a solutal flux intensity factor $\mathcal{F}(t)$. The latter measures the intensity of the solute flux in the dendrite tip region and can be calculated by contour (2D) or surface (3D) integration around the tip of each needle. We first present an extended formulation of the 2D DNN model where needles have a finite thickness and parabolic tips. This formulation remains valid for a larger range of tip Péclet number than the original thin needle formulation and is readily extended to 3D needles with paraboloidal tips. The 3D DNN model based on this thick-needle formulation is developed for both isothermal and directional solidification. Model predictions are validated by comparisons with known analytical solutions that describe the early transient and steady-state growth regimes. We exploit the power of the DNN model to characterize the competitive growth of well-developed secondary branches in $3 \mathrm{D}$ on the scale of the diffusion length. The results show that the length of active secondary branches increases as a power law of distance behind the tip with an exponent in good quantitative agreement with experimental measurements. Finally, we apply the model to simulate the three-dimensional directional solidification of an $\mathrm{Al}-7 \mathrm{wt} \% \mathrm{Si}$ alloy, which we directly compare to observed microstructures from microgravity experiments onboard the International Space Station. The predictions of selected microstructural features, such as dendrite arm spacings, show a good agreement with experiments. The computationally-efficient DNN model opens new avenues for investigating the dynamics of large dendritic arrays at length and time scales relevant to solidification experiments and processes.
\end{abstract}


Keywords: solidification, dendritic growth, microstructure, multiscale modeling, alloy.

\section{Introduction}

Dendritic microstructures are the most common among cast metals and alloys [1, 2]. The geometrical features of these structures arise from a subtle interplay between microscopic interfacial phenomena and macroscopic solute and heat transport, and are crucial to mechanical properties of a cast part, and subsequently to its performance during service $[1,3,4]$. Within a grain, interactions among individual dendritic branches determine its inner structure, such as the primary spacing crucial for the mechanical strength of a dendritic grain. In polycrystalline microstructures too, long range interactions between the growing dendrites play a key role in shaping the grain structure that critically influences the mechanical properties of a material. Hence, our ability to understand, predict, and control the microstructure selection mechanisms across all critical length and time scales is key to develop innovative materials and processes.

At the scale of an entire solidification process or experiment, computational approaches include continuum models $[5,6,7,8]$, models based on dynamics of average dendritic grain enveloppes $[9,10,11,12,13]$, and approaches coupling cellular automata with finite elements [14, 15, 16], finite differences [17], or Lattice Boltzmann methods $[18,19]$. This type of models can be used at the scale of a dendritic array to investigate mechanisms of intragrain [17] or intergrain [20] microstructure selection up to the scale of entire casting experiments, thus providing average statistical predictions of microstructure selection by complex phenomena such as the columnar-to-equiaxed transition [21]. However, those volume-averaged equilibrium-based models do not resolve quantitatively the transient interactions between individual dendritic branches that are crucial to dendritic microstructure selection.

At a smaller scale, the phase-field (PF) approach is the method of choice to quantitatively simulate complex solid-liquid interface patterns [22]. The theoretical development of the thin-interface limit [23, 24] and the anti-trapping current for alloy solidification [25, 26, 27], combined with advanced numerical techniques such as adaptive meshing $[28,29]$ and massive parallelization [30, 31], now enable quantitative PF predictions at the scale of spatially extended three-dimensional arrays of cells [32, 33] and dendrites [34, 35]. However, quantitative predictions of dendritic growth dynamics with PF require an accurate morphological description of each dendrite tip, which makes simulations extremely challenging for concentrated alloys that usually solidify as a hierarchical network of thin branches with several orders of magnitude separating the scale of a dendrite tip radius and the larger scale of diffusive transport in the melt.

In order to bridge the scale gap between phase-field and coarse-grained models, we recently developed a multiscale Dendritic Needle Network (DNN) approach that quantitatively predicts the dynamics of individual

\footnotetext{
${ }^{*}$ Corresponding author

Email addresses: dtourret@lanl.gov (D. Tourret), a.karma@neu.edu (A. Karma)
} 
branches in complex dendritic networks during alloy solidification at a scale much larger than the diffusion length. This model, first developed in 2D [36, 37], is rigorously valid as long as the dendrite tip Péclet number remains relatively small, which is the case for many common processing conditions. Hence, the approach is well suited to describe concentrated alloys where dendrites form hierarchical tree-like structures with several generations of needle-like branches.

In this approach, a dendritic grain, such as the crystal in Fig. 1a [38], is modeled as a network of thin needles, as in Fig. 1b. The dynamics of each needle tip is prescribed by two conditions that jointly determine the evolution of its velocity $V(t)$ and radius $\rho(t)$.

The first condition is a solute conservation equation formulated at an intermediate scale much larger than the dendrite tip radius $\rho$ and much smaller than the diffusion length $D / V$, with $D$ the solute diffusion coefficient in the liquid (Fig. 1c). In 2D, this condition links the product $\rho V^{2}$ to a flux intensity factor $\mathcal{F}(t)$ that measures the strength of the incoming solute flux at the tip [36]. The value of $\mathcal{F}(t)$ can be calculated using a contour integral around the tip, e.g. in Ref. [36] using the J-integral classically used in fracture mechanics to compute stress intensity factors at the tip of a crack [40].

The second condition, formulated at the scale of the dendrite tip (Fig. 1d), is a standard microscopic solvability condition for the existence of a solution to the shape-preserving growth of a parabola/paraboloid. It relates the product $\rho^{2} V$ to the strength of surface tension anisotropy [41, 42, 43]. While $\rho^{2} V$ remains constant in the DNN model, the flux intensity factor $\mathcal{F}(t)$ at each needle tip evolves with the surrounding solutal field, hence enabling the approach to capture long-range diffusive interactions between branches in both transient and steady-state growth regimes.

The DNN model derives its efficiency from the fact that the solid-liquid interface is not explicitly tracked and that the diffusion field can be discretized on a scale comparable to or larger than $\rho$. In comparison, phase-field typically requires a computational grid size one order of magnitude smaller than the tip radius for a reliable morphological description of the dendrite tip and hence of its growth dynamics [24, 44]. This leads to DNN simulations faster than PF simulations by four to five orders of magnitude in 2D and 3D, respectively, if both models are discretized on a finite-difference grid using an explicit time stepping.

In this article, we present a three-dimensional formulation of the DNN model. In Section 2, we summarize the sharp interface solidification problem and the two-dimensional model as presented in Ref. [36]. Then, we propose a new 2D formulation for thick branches with parabolic tips. This thick-needle formulation has the dual benefit that it increases the range of tip Péclet number where the DNN approach is quantitatively valid and that it can be readily extended to $3 \mathrm{D}$ where branches have paraboloidal tips. We provide details of our first numerical implementation in Section 3. In Section 4, we validate the DNN predictions through comparisons with analytical solutions in a steady-state growth regime and with an analytical scaling law for the early stage transient growth of an equiaxed 3D grain with six branches growing along principal crystal axes. In Section 5, we compare the predicted dynamics of secondary sidebranches against experimental 
measurements of dendritic envelope shapes. Then, in Section 6, we apply the DNN model to a fully threedimensional directional solidification experiment of an $\mathrm{Al}-7 \mathrm{wt} \% \mathrm{Si}$ alloy, and directly compare the results to microgravity experiments performed in the framework of the CETSOL project $[45,46,47]$ (Columnarto-Equiaxed Transition in SOLidification Processing). Finally, in Section 7, we summarize our results and discuss further investigations made possible by this new modeling approach.

\section{Modeling}

\subsection{Sharp-interface solidification model}

We consider the solidification of a binary alloy in a purely diffusive regime with negligible diffusion in the solid phase, such that the solute concentration $c$ in the liquid follows the diffusion equation

$$
\frac{\partial c}{\partial t}=D \nabla^{2} c .
$$

The solid-liquid interface evolves with a normal velocity $v_{n}$ given by mass conservation at the interface, i.e. by the Stefan condition

$$
(1-k) c_{l} v_{n}=-\left.D \frac{\partial c}{\partial n}\right|_{i}
$$

where $k$ is the solute partition coefficient at the interface, $c_{l}$ the concentration at the interface in the liquid, and $\partial c /\left.\partial n\right|_{i}$ is the normal solute concentration gradient at the interface in the liquid.

Thermal diffusion is considered to be much faster than solute diffusion, so that the surrounding temperature $T$ can be fixed, for instance at $T=T_{0}$ for isothermal solidification. Thus, the concentration at the interface $c_{l}$ is determined by local thermodynamic equilibrium, i.e. by the Gibbs-Thomson relation

$$
T=T_{M}+m c_{l}-\Gamma_{s l} f(\theta) \kappa,
$$

with $T_{M}$ the melting temperature of the pure solvent, $m$ the liquidus slope (with $m<0$ ), $\Gamma_{s l}$ the GibbsThomson coefficient of the interface, and $\kappa$ the interface curvature. The term $f(\theta)$ models the dependence of the interface stiffness $\gamma+\gamma_{\theta \theta}$ on the orientation $\theta$, where $\gamma$ is the excess free-energy of the solid-liquid interface and $\gamma_{\theta \theta}$ denotes the second derivative of $\gamma$ with respect to $\theta$.

We reformulate Eqs. (1), (2) and (3) as a function of a reduced solute field

$$
u \equiv \frac{c_{l}^{0}-c}{(1-k) c_{l}^{0}},
$$

with $c_{l}^{0}=\left(T_{M}-T_{0}\right) /|m|$ the liquid equilibrium composition for a flat interface at a reference temperature $T_{0}$. Thus, the problem reduces to

$$
\begin{aligned}
\frac{\partial u}{\partial t} & =D \nabla^{2} u, \\
{\left[1-(1-k) u_{i}\right] v_{n} } & =\left.D \frac{\partial u}{\partial n}\right|_{i}, \\
u_{i} & =d_{0} f(\theta) \kappa,
\end{aligned}
$$


with $u_{i}=\left(c_{l}^{0}-c_{l}\right) /\left[(1-k) c_{l}^{0}\right]$ the value of $u$ at the interface and $d_{0}=\Gamma_{s l} /\left[|m|(1-k) c_{l}^{0}\right]$ the solute capillary length at $T_{0}$. The condition $c \rightarrow c_{\infty}$ far from the solid becomes $u \rightarrow \Omega$ with the solute supersaturation

$$
\Omega \equiv \frac{c_{l}^{0}-c_{\infty}}{(1-k) c_{l}^{0}} .
$$

\subsection{Dendritic needle network model for isothermal solidification}

At a low supersaturation $\Omega \ll 1$ relevant to most castings and experiments, crystal structures usually grow in the form of a network of thin needle-like dendritic branches, like the crystal illustrated in Fig. 1a. In this case, the DNN model [36] represents a dendritic grain as a network of sharp line segments at local equilibrium, like in Fig. 1b. The growth dynamics of each branch can be formulated by combining two independent conditions at two distinct length scales: (i) a mass conservation condition at an intermediate scale between the tip radius $\rho$ and the diffusion length $D / V$ (Fig. 1c), and (ii) a solvability condition at the scale of $\rho$ (Fig. 1d). These two conditions, respectively summarized in Sections 2.2.2 and 2.2.1, uniquely prescribe the instantaneous tip radius $\rho(t)$ and tip velocity $V(t)$ of each individual dendritic branch.

\subsubsection{Solvability condition at the tip}

Numerous studies $[41,42,43,48]$ have established that the sharp interface problem, Eqs. (5)-(7), neglecting the small capillary correction in Eq. (6) such that $v_{n} \approx D \partial u /\left.\partial n\right|_{i}$, admits a steady solution only if

$$
\rho^{2} V=\frac{2 D d_{0}}{\sigma}
$$

where the tip selection parameter $\sigma$ is uniquely fixed by the strength of crystalline anisotropy. This solvability theory was validated by phase-field simulations, e.g. [23, 28, 49]. Furthermore, since the solute field relaxes on the tip scale in a time $\sim \rho^{2} / D$, which is much smaller than the diffusive time $\sim D / V^{2}$, the product $\rho^{2} V$ relaxes to a constant value early during the transient development of a dendrite, while both $\rho(t)$ and $V(t)$ still exhibit significant time variations [49]. Hence, this allows us to consider Eq. (9) valid in the early transient growth regime of each needle.

\subsubsection{Solute flux intensity around the tip}

At a scale much larger than the tip radius $\rho$, surface tension effects can be neglected and the dendritic branches can essentially be considered as infinitely sharp line segments at the equilibrium concentration, i.e. $u=0$ at $T=T_{0}$. Also, at a scale much smaller than the diffusion length $D / V$, the solute field relaxes sufficiently fast to assume a Laplacian $u$ field in the vicinity of a needle tip. Yet, in two dimensions, the solution of $\nabla^{2} u=0$ with $u=0$ on a sharp needle exhibits a square root singularity of the normal solute gradient in the vicinity of the tip such that [50]

$$
\left.\frac{\partial u}{\partial y}\right|_{y=0}=\frac{\mathcal{F}}{\sqrt{d_{0}\left(x_{t}-x\right)}} \text { when } x \rightarrow x_{t}
$$


where $+x$ is the needle growth direction, and $x=x_{t}$ and $y=0$ are the tip coordinates.

Additionally, on a scale $\gg \rho$, the region behind the tip may be considered quasi-planar, so that the normal velocity of the solid-liquid interface follows

$$
v_{n}=\frac{\mathrm{d} y_{i}(t)}{\mathrm{d} t}=\left.D \frac{\partial u}{\partial y}\right|_{y=y_{i}(t)},
$$

where $y_{i}(t)$ is the interface position along the $y$ axis perpendicular to the needle. Since $y_{i}(t) \ll D / V$ for $\Omega \ll 1$, the last term $\partial u / \partial y$ can be evaluated at $y=0$ instead of $y=y_{i}(t)$, which yields

$$
\left.\frac{\mathrm{d} y_{i}(t)}{\mathrm{d} t} \approx D \frac{\partial u}{\partial y}\right|_{y=0}=\frac{D \mathcal{F}}{\sqrt{d_{0}\left(x_{t}-x\right)}} .
$$

At this scale, $x_{t}-x \ll D / V$, and one can assume that the interface grows in a quasi steady-state, i.e. with very slow variations of the flux intensity factor $\mathcal{F}$ on a diffusive time scale. Hence, one can apply the change of variable $x_{t}-x=V t$, and integrate Eq. (12) to find the shape $y_{i}(x)$ of the needle

$$
y_{i}(x)=2 \frac{D \mathcal{F}}{V} \sqrt{\frac{x_{t}-x}{d_{0}}} .
$$

This is the equation of a parabola $x=x_{t}-y^{2} /(2 \rho)$ with a tip radius $\rho=2(\mathcal{F} D / V)^{2} / d_{0}$, which yields

$$
\rho V^{2}=\frac{2 D^{2} \mathcal{F}^{2}}{d_{0}} .
$$

The combination of Eqs. (9) and (14) prescribes the growth conditions $\rho$ and $V$ of a needle. While the solvability condition, Eq. (9), imposes a constant value of $\rho^{2} V$, the flux condition, Eq. (14), accounts for the evolution of the long range diffusive field seen by the tip, by introducing the time-dependent flux intensity factor $(\mathrm{FIF}) \mathcal{F}(t)$, defined as

$$
\left.\mathcal{F}(t) \equiv \lim _{x \rightarrow x_{t}} \sqrt{d_{0}\left(x_{t}-x\right)} \frac{\partial u(x, y, t)}{\partial y}\right|_{y=0}
$$

In $2 \mathrm{D}$, we have shown [36] that the FIF may be calculated exactly using a contour integral similar to the J-integral commonly used in elasticity to calculate the stress intensity factors in the vicinity of a crack tip [40], and also used in various problems involving a Laplacian field, e.g. in the case of viscous fingering [51]. Using the mathematical isomorphism between mode III pure antiplane shear fracture and Laplacian diffusion, we can write [36]

$$
\mathcal{F}^{2}=\frac{d_{0}}{2 \pi} \int_{\Gamma}\left\{\left[\left(\frac{\partial u}{\partial x}\right)^{2}-\left(\frac{\partial u}{\partial y}\right)^{2}\right] n_{x}+2 \frac{\partial u}{\partial x} \frac{\partial u}{\partial y} n_{y}\right\} \mathrm{d} \Gamma
$$

along an open contour $\Gamma$ surrounding the tip, as in Fig. 2a, where $\left(n_{x}, n_{y}\right)$ are the coordinates of the normal $\mathbf{n}$ to $\Gamma$. 


\subsubsection{Summary of the 2D isothermal model with sharp branches}

In summary, the solute field in the liquid follows

$$
\frac{\partial u}{\partial t}=D \nabla^{2} u
$$

with the boundary conditions

$$
\begin{array}{ll}
u=0 & \text { on the needles, } \\
u=\Omega & \text { far away, }
\end{array}
$$

while the dynamics of each needle in the network is obtained by combining the tip flux condition, Eq. (14), with the solvability condition, Eq. (9), hence yielding

$$
\begin{aligned}
\frac{\rho(t)}{d_{0}} & =\left(2 / \sigma^{2}\right)^{1 / 3} \mathcal{F}(t)^{-2 / 3}, \\
\frac{V(t) d_{0}}{D} & =(2 \sigma)^{1 / 3} \mathcal{F}(t)^{4 / 3},
\end{aligned}
$$

where only $V(t)$ is required for the needle network to evolve. The FIF at each needle tip can be calculated by the integration of Eq. (16) along an open contour $\Gamma$ surrounding the needle tip (Fig. 2a).

Additionally, the position of the solid-liquid interface can be approximately reconstructed using the quasione-dimensional form of the Stefan condition on the side of a needle with the normal gradient evaluated on the needle,

$$
\left.\frac{\mathrm{d} y_{i}(t)}{\mathrm{d} t} \approx D \frac{\partial u}{\partial y}\right|_{y=0} .
$$

This description of the DNN model corresponds to the one presented in Ref. [36], which is here referred to as the sharp needle formulation of the model. Because in three dimensions there is no analytical solution for a Laplacian field around a sharp tip at a fixed concentration, such as Eq. (10) in 2D, this formulation of the model is not possible to directly extend to 3D. Thus, in the following subsection, we propose an alternative thick needle formulation of the model in $2 \mathrm{D}$ that accounts for a finite thickness of a needle. This formulation extends the quantitative validity of the approach to larger tip Péclet number and can be directly extended to 3D.

\subsection{Extension to a parabolic needle}

Let us consider, as schematized in Fig. 2b, a parabolic needle tip of radius $\rho$ growing at a velocity $V$ in the $+x$ direction, with $u=0$ on the interface location, i.e. along $y_{i}(x)=\sqrt{2 \rho\left(x_{t}-x\right)}$. At the corresponding time scale, one may assume that the growth of the needle tip is quasi-stationary and that the parabolic tip shape is preserved in a moving frame progressing at a velocity $V$ in the needle growth direction. Hence, the solute flux normal to the solid-liquid interface $D \partial u / \partial n \approx v_{n}$ only contributes to its advancing in the main 
growth direction at a velocity $V$. The integration of the solute conservation at the interface in the tip region over a length $a$, i.e. over a contour $\Gamma^{\prime}$ between $x=x_{t}-a$ and $x=x_{t}$ as in Fig. $2 \mathrm{~b}$, yields

$$
D \int_{\Gamma^{\prime}} \frac{\partial u}{\partial n} \mathrm{~d} \Gamma^{\prime}=\int_{-y_{a}}^{y_{a}} V \mathrm{~d} y=2 V \sqrt{2 \rho a},
$$

with $y_{a} \equiv y_{i}\left(x_{t}-a\right)=\sqrt{2 \rho a}$. Thus, we can write

$$
\rho V^{2}=\frac{1}{8 a}\left(D \int_{\Gamma^{\prime}} \frac{\partial u}{\partial n} \mathrm{~d} \Gamma^{\prime}\right)^{2},
$$

such that, if we define the flux intensity factor as

$$
\mathcal{F} \equiv \frac{1}{4 \sqrt{a / d_{0}}} \int_{\Gamma^{\prime}} \frac{\partial u}{\partial n} \mathrm{~d} \Gamma^{\prime},
$$

the product $\rho V^{2}$ follows a similar law as Eq. (14),

$$
\rho V^{2}=\frac{2 D^{2} \mathcal{F}^{2}}{d_{0}}
$$

In order to integrate the value of $\mathcal{F}(t)$, now defined by Eq. (25), we write Laplace equation in a moving frame of velocity $V$,

$$
D \nabla^{2} u=-V \frac{\partial u}{\partial x} .
$$

Then, if we integrate $\nabla^{2} u$ over a surface $\Sigma$ bounded by the contours $\Gamma$ and $\Gamma^{\prime}$ as in Fig. 2b, we can use the divergence theorem to write

$$
\begin{aligned}
\int_{\Gamma^{\prime}} \frac{\partial u}{\partial n} \mathrm{~d} \Gamma^{\prime}-\int_{\Gamma} \frac{\partial u}{\partial n} \mathrm{~d} \Gamma & =-\iint_{\Sigma} \nabla^{2} u \mathrm{~d} \Sigma \\
& =\frac{V}{D} \iint_{\Sigma} \frac{\partial u}{\partial x} \mathrm{~d} \Sigma,
\end{aligned}
$$

where, in order to be consistent with the sharp needle model, we choose to have the normal $\mathbf{n}$ pointing outward of the closed contour $\left(\Gamma+\Gamma^{\prime}\right)$ along $\Gamma$ (see Fig. 2a), while pointing outward of the dendrite, i.e. inward the contour, along $\Gamma^{\prime}$ (see Fig. 2b), which justifies the minus sign in the left hand side of Eq. (28). Hence, the FIF can be calculated from Eq. (25) where the integral along $\Gamma^{\prime}$ is given by

$$
\int_{\Gamma^{\prime}} \frac{\partial u}{\partial n} \mathrm{~d} \Gamma^{\prime}=\int_{\Gamma} \frac{\partial u}{\partial n} \mathrm{~d} \Gamma+\frac{V}{D} \iint_{\Sigma} \frac{\partial u}{\partial x} \mathrm{~d} \Sigma
$$

using any contour $\Gamma$ joining the needle at $x=x_{t}-a$.

The resulting thick needle DNN model follows the same growth laws as the sharp needle model, with a constant value of $\rho^{2} V$ and a product $\rho V^{2} \sim \mathcal{F}(t)^{2}$. The only differences are the new definition of the flux intensity factor $\mathcal{F}(t)$, Eq. (25), where the integral term of the flux over the contour $\Gamma^{\prime}$ can be integrated over a chosen outer contour $\Gamma$ using Eq. (29), as well as imposing $u$ at equilibrium on a parabolic interface instead of a sharp line segment. 
Alternatively, the limit of this formulation for a vanishingly thin needle can be used to replace the calculation of $\mathcal{F}$ through the J-integral, Eq. (16), in a model with a sharp needle. In this case, the integration contour can also be taken vanishingly small with $\Gamma^{\prime}=\Gamma$, such that the flux intensity factor defined by Eq. (25) can be directly integrated along the contour $\Gamma^{\prime}$ by integration of the lateral flux normal to the sharp needle between $x=x_{t}$ and $x=x_{t}-a$, together with the contribution in the $x$-direction facing the growth of the needle at the tip location. This alternative thin-needle formulation has the advantages of not requiring the definition of an outer contour $\Gamma$ but only the choice of an integration distance $a$, and it does not include a velocity-dependent correction (i.e. the surface interval over $\Sigma$ ). It is therefore less likely to show results variations with the choice of this integration contour $\Gamma$. We compare these various $2 \mathrm{D}$ formulations in Section 4.1 .

\subsection{Extension to three dimensions}

The DNN model with parabolic branches can be directly extended to a three-dimensional dendrite growing as a paraboloid of revolution with a circular cross section of radius $r_{i}(x)=\sqrt{2 \rho\left(x_{t}-x\right)}$.

In $3 \mathrm{D}, \Gamma$ and $\Gamma^{\prime}$ are surfaces joining at the intersection of $\Gamma$ with the needle at $x=x_{t}-a$, and $\Sigma$ is the volume enclosed within the closed surface $\left(\Gamma+\Gamma^{\prime}\right)$. Similarly as in $2 \mathrm{D}$, assuming a shape-preserving paraboloid growing in a quasi-stationary regime at a velocity $V$, the mass conservation $D \partial u / \partial n \approx v_{n}$ along the paraboloid surface up to a length $a$ behind the tip yields

$$
D \iint_{\Gamma^{\prime}} \frac{\partial u}{\partial n} \mathrm{~d} \Gamma^{\prime}=\int_{0}^{2 \pi} \int_{0}^{r_{a}} V r \mathrm{~d} r \mathrm{~d} \theta=2 \pi a \rho V,
$$

with $r_{a}=\sqrt{2 \rho a}$ the cross-section radius of the paraboloid at $x=x_{t}-a$. Thus, we can define the flux intensity factor

$$
\mathcal{F} \equiv \frac{1}{2 \pi a} \iint_{\Gamma^{\prime}} \frac{\partial u}{\partial n} \mathrm{~d} \Gamma^{\prime}
$$

and write the flux condition at the tip

$$
\rho V=D \mathcal{F}
$$

Similarly as in two dimensions, the flux over the surface $\Gamma^{\prime}$ can be calculated over any surface $\Gamma$ that intersects the paraboloid at $x=x_{t}-a$ using

$$
\iint_{\Gamma^{\prime}} \frac{\partial u}{\partial n} \mathrm{~d} \Gamma^{\prime}=\iint_{\Gamma} \frac{\partial u}{\partial n} \mathrm{~d} \Gamma+\frac{V}{D} \iiint_{\Sigma} \frac{\partial u}{\partial x} \mathrm{~d} \Sigma
$$

The growth conditions $(\rho, V)$ of a needle tip can thus be obtained by combining the new flux balance, Eq. (32), with the solvability condition

$$
\rho^{2} V=\frac{2 D d_{0}}{\sigma}
$$

which is similar to the $2 \mathrm{D}$ condition, only with a tip selection parameter $\sigma$ that follows a different law with respect to the strength of surface tension anisotropy as compared to $2 \mathrm{D}$ [43]. 


\subsection{Non-isothermal solidification}

For non-isothermal conditions, one needs to set a reference temperature $T_{0}$, here chosen as the solidus temperature of the alloy. In the case of directional solidification, the temperature profile is approximated as

$$
T=T_{0}+G\left(x-V_{p} t\right)
$$

with a given temperature gradient $G$ and pulling velocity $V_{p}$, both oriented along the $x$ axis. The GibbsThomson condition, Eq. (3), hence becomes

$$
\frac{c_{l}}{c_{l}^{0}}=1-(1-k) d_{0} f(\theta) \kappa-(1-k) \frac{x-V_{p} t}{l_{T}}
$$

where $c_{l}^{0}=c_{\infty} / k$ is the liquid equilibrium composition for a flat interface at the solidus temperature $T_{0}$ with $c_{\infty}$ the nominal composition of the alloy, $d_{0} \equiv \Gamma /\left[|m|(1-k) c_{l}^{0}\right]$ remains the capillarity length at $T_{0}$, and $l_{T} \equiv\left[|m|(1-k) c_{l}^{0}\right] / G$ is the thermal length, which is the length over which the temperature variation induced by the gradient $G$ equals the freezing range of the alloy. As a function of the reduced solute field $u$ from Eq. (4), the Gibbs-Thomson condition, Eq. (7), becomes

$$
u_{i}=d_{0} f(\theta) \kappa+\frac{x-V_{p} t}{l_{T}} .
$$

At a scale $\gg \rho$, where the curvature along the side of the needles can be neglected, the equilibrium along the interface is

$$
u_{i} \approx \frac{x-V_{p} t}{l_{T}},
$$

varying linearly from $u_{i}=0$ at the solidus to $u_{i}=1$ at the liquidus temperature, with a far-field condition $c \rightarrow c_{\infty}$ expressed as $u(x \rightarrow+\infty)=1$.

At the intermediate scale of Fig. 1c and Fig. 2, also much smaller than the thermal length $l_{T}$, the value of $u_{i}$ remains close to its value at the tip location, $u_{t}$. Then, while the prefactor $\left[1-(1-k) u_{i}\right]$ in the Stefan condition, Eq. (6), can be neglected in isothermal conditions due to $u_{i} \approx 0$, it appears as prefactor of $v_{n}$ in directional solidification as

$$
\left[1-(1-k) u_{t}\right] v_{n}=\left.D \frac{\partial u}{\partial n}\right|_{i} .
$$

The solvability condition, Eq. (9) in 2D and Eq. (34) in 3D, can thus be derived identically as in isothermal conditions, only replacing $d_{0}$ by a temperature dependent capillarity length $d_{0}^{*}=d_{0} /\left\{1-(1-k) u_{t}\right\}$, since $d_{0}$ is now defined at the reference (solidus) temperature $T_{0}[36,37]$, yielding

$$
\rho^{2} V=\frac{1}{1-(1-k) u_{t}} \frac{2 D d_{0}}{\sigma},
$$

Additionally, using $u_{i} \approx u_{t}$ along and inside the integration contour or surface $\Gamma$, one can derive Eq. (14) or (26) identically as in Sections 2.2 .2 and 2.3, only keeping the $\left[1-(1-k) u_{t}\right]$ prefactor to $v_{n}$ and hence $V$ 
in the Stefan condition, Eq. (39) [36, 37], yielding

$$
\begin{aligned}
\rho V^{2} & =\frac{1}{\left[1-(1-k) u_{t}\right]^{2}} \frac{2 D^{2} \mathcal{F}^{2}}{d_{0}} & & \text { (in } 2 \mathrm{D}) \\
\rho V & =\frac{1}{\left[1-(1-k) u_{t}\right]} D \mathcal{F} & & \text { (in } 3 \mathrm{D}) .
\end{aligned}
$$

In summary, the model for directional solidification combines the diffusion equation in the liquid, $\partial u / \partial t=$ $D \nabla^{2} u$, with imposed boundary conditions

$$
\begin{array}{rlr}
u_{i} & =\frac{x-V_{p} t}{l_{T}} & \text { on the needles, } \\
u=1 & \text { at } x \rightarrow \infty,
\end{array}
$$

with the growth of each needle obtained by combining the solvability condition, Eq. (40), and the conservation Eq. (41) in $2 \mathrm{D}$, or $(42)$ in $3 \mathrm{D}$, where $\mathcal{F}(t)$ is integrated with Eq. (16) or (25) in 2D, or with Eq. (31) in $3 \mathrm{D}$.

\subsection{Sidebranching}

Similarly as in Ref. [36], sidebranches are generated periodically at a distance $d=(N+\delta N) \rho$ behind a needle tip of radius $\rho$ each time the needle grows by a distance $d$, with $\delta N$ a random fluctuation in the range $[-\Delta N / 2 ; \Delta N / 2]$. The initial length of a sidebranch is $\sqrt{2 \rho d}$. The frequency of sidebranching events $N$ and its fluctuation amplitude $\Delta N$ are input parameters to the model, chosen to match experimental observations, usually showing sidebranches appearing at distance of about five to ten tip radii behind the parent tip $[38,39]$. We have shown that as long as sidebranching is frequent enough to seed the competitive growth of sidebranches, the exact choice of model parameters $N$ and $\Delta N$ has little influence on the final selected microstructures [36].

\section{Numerical implementation}

\subsection{Scaling}

In order to numerically solve the DNN equations, we chose to scale space and time using using the theoretical steady-state tip radius $\rho_{s}$ and velocity $V_{s}$, like in Ref. [36]. Hence, in the scaled coordinate system $(\tilde{x}, \tilde{y}, \tilde{z})$ with $\tilde{x}=x / \rho_{s}, \tilde{y}=y / \rho_{s}$, and $\tilde{z}=z / \rho_{s}$, the diffusion equation in the liquid becomes

$$
\frac{\partial u}{\partial \tilde{t}}=\widetilde{D} \nabla^{2} u
$$

with $\tilde{t} \equiv t V_{s} / \rho_{s}$ and $\widetilde{D} \equiv D /\left(\rho_{s} V_{s}\right)$. 
In isothermal solidification in $2 \mathrm{D}$, the evolution of the scaled tip radius $\tilde{\rho} \equiv \rho / \rho_{s}$ and velocity $\widetilde{V} \equiv V / V_{s}$ of a needle thus follows

$$
\begin{aligned}
& \tilde{\rho}^{2} \widetilde{V}=1 \\
& \tilde{\rho} \widetilde{V}^{2}=2 \widetilde{D}^{2} \widetilde{\mathcal{F}}^{2}
\end{aligned}
$$

with a flux intensity factor given by

$$
\widetilde{\mathcal{F}}^{2}=\frac{1}{2 \pi} \int_{\Gamma}\left\{\left[\left(\frac{\partial u}{\partial \tilde{x}}\right)^{2}-\left(\frac{\partial u}{\partial \tilde{y}}\right)^{2}\right] n_{x}+2 \frac{\partial u}{\partial \tilde{x}} \frac{\partial u}{\partial \tilde{y}} n_{y}\right\} \mathrm{d} \Gamma
$$

in the sharp needle formulation [36], or by

$$
\widetilde{\mathcal{F}}=\frac{1}{4 \sqrt{\tilde{a}}} \int_{\Gamma^{\prime}} \frac{\partial u}{\partial n} \mathrm{~d} \Gamma^{\prime}
$$

for a parabolic needle, with $\tilde{a} \equiv a / \rho_{s}$. In 3D, the mass conservation equation at the tip, Eq. (47), becomes

$$
\tilde{\rho} \widetilde{V}=\widetilde{D} \widetilde{\mathcal{F}}
$$

with

$$
\widetilde{\mathcal{F}}=\frac{1}{2 \pi \tilde{a}} \iint_{\Gamma^{\prime}} \frac{\partial u}{\partial n} \mathrm{~d} \Gamma^{\prime}
$$

For directional solidification, the growth dynamics of each needle is given by $[36,37]$

$$
\begin{aligned}
\tilde{\rho}^{2} \widetilde{V} & =\frac{1-(1-k) u_{s}}{1-(1-k) u_{t}} \\
\tilde{\rho} \widetilde{V}^{2} & =\frac{2 \widetilde{D}^{2} \widetilde{\mathcal{F}}^{2}}{\left[1-(1-k) u_{t}\right]^{2}} \\
\tilde{\rho} \widetilde{V} & =\frac{2 \widetilde{D} \widetilde{\mathcal{F}}}{1-(1-k) u_{t}}
\end{aligned}
$$

where $u_{s}$ is the value of $u_{t}$ at the the steady-state for a tip of radius $\rho_{s}$ and velocity $V_{s}$.

In order to perform such a scaling, one needs to a priori determine steady-state growth conditions $\left(\rho_{s}, V_{s}\right)$ in isothermal solidification, and $\left(\rho_{s}, u_{s}\right)$ in directional solidification (since in the latter case $\left.V_{s}=V_{p}\right)$. This can be done by combining the steady-state solvability condition

$$
\rho_{s}^{2} V_{s}=\frac{1}{1-(1-k) u_{s}} \frac{2 D d_{0}}{\sigma}
$$

with a relation linking the steady-state tip Péclet number $P \equiv \rho_{s} V_{s} /(2 D)$ to the local solute supersaturation $\Omega_{s}$ at the tip of an isolated free dendrite. This additional relation between $P$ and $\Omega_{s}$ can be freely chosen, but the most natural choice is the analytical Ivantsov solution $[52,3]$

$$
\begin{aligned}
& \Omega_{s}=\sqrt{\pi P} \exp (P) \operatorname{erfc}(\sqrt{P}) \\
& \Omega_{s}=P \exp (P) \mathrm{E}_{1}(P)
\end{aligned}
$$


where

$$
\operatorname{erfc}(\eta) \equiv \frac{2}{\sqrt{\pi}} \int_{\eta}^{\infty} \exp \left(-\tau^{2}\right) \mathrm{d} \tau
$$

and

$$
\mathrm{E}_{1}(\eta) \equiv \int_{\eta}^{\infty} \frac{\exp (-\tau)}{\tau} \mathrm{d} \tau
$$

In two dimensions, an alternative choice is the exact solution for a sharp needle, i.e. the approximation of the Ivantsov solution, Eq. (56), for $\Omega \ll 1$

$$
\Omega_{s}=\sqrt{\pi P} .
$$

In isothermal solidification, by definition $\Omega_{s}$ is the imposed supersaturation $\Omega$, while in directional solidification, we use the definition of $u_{s}$ as a function of the steady-state tip solute concentration $c_{s}$,

$$
u_{s}=\frac{c_{l}^{0}-c_{s}}{(1-k) c_{l}^{0}}
$$

to write

$$
\Omega_{s} \equiv \frac{c_{s}-k c_{l}^{0}}{(1-k) c_{s}}=\frac{1-u_{s}}{1-(1-k) u_{s}}
$$

and numerically solve the system of three equations, Eq. (55), Eq. (62), and the chosen relation between $P$ and $\Omega_{s}$, e.g. Eq. (56) in $2 \mathrm{D}$ or Eq. (57) in 3D.

In our simulations, the 2D sharp needle model is scaled using Eq. (60) (i.e. reducing to the formulation from Ref. [36]), the thick needle model is scaled using Eq. (56) in 2D, and using Eq. (57) in 3D. These scalings all yield $\tilde{\rho} \rightarrow 1$ and $\widetilde{V} \rightarrow 1$ for an isolated free dendrite. It is worth mentioning that the sharp 2D model could be scaled using Eq. (56), just like the parabolic 2D model could be scaled using Eq. (60), which would only lead to $\tilde{\rho}$ and $\widetilde{V}$ not tending to one for an isolated free dendrite at the steady-state, but still conserving $\tilde{\rho}^{2} \widetilde{V}=1$. Other scalings are of course possible, e.g. using alloy parameters $D$ and $d_{0}$.

\subsection{Implementation}

We solve the equations of the DNN model on a finite difference grid of regular square (cubic) elements of dimension $\Delta x$, using an explicit Euler time stepping scheme with a constant time step $\Delta t$, equal to $\Delta x^{2} /(4 D)$ in $2 \mathrm{D}$, and $\Delta x^{2} /(6 D)$ in 3D. Both the evolution of the diffusive solute field $u$ from Eq. (45) and that of the growth conditions $\{\rho(t), V(t)\}$ of each needle from Eqs. (52)-(54) are thus solved explicitly. For the latter, in order to keep the time stepping purely explicit, the velocity correction term in the calculation of the flux intensity factor $\mathcal{F}(t)$ uses the tip velocity $V(t)$ at the previous time step in Eq. (29) or (33) (in $2 \mathrm{D}$ and $3 \mathrm{D}$, respectively).

We implemented the model for massively parallel calculation on Graphic Processing Units (GPUs) with the Compute Unified Device Architecture (CUDA), using a single GPU per simulation. The time loop essentially consists of three kernels, respectively performing the following steps: (i) diffusion of solute in the liquid, (ii) growth of the needles, and (iii) generation of sidebranches. For the diffusion step, the domain is 
divided in contiguous sections of block size $16 \times 32$ threads in $2 \mathrm{D}$, and $8 \times 8 \times 8$ threads in $3 \mathrm{D}$. The growth step, which consists of the integration of $\mathcal{F}(t)$ and the updating of $u=u_{i}$ along the needle interface with the new needle length and tip radius, is parallelized such that each thread corresponds to one needle. The sidebranching step is also performed on the GPU, but not parallelized (i.e. using only one thread), so as to ensure that creating new branches and storing their properties in an array proceeds in an orderly manner. (This part could be parallelized more efficiently, as long as a sufficient care is taken to avoid so-called race conditions when creating new branches and storing them into the needle array.) An optional step occurs periodically in order to shift the entire domain in the $x$ direction, so that the most advanced tip remains at the same position in the simulation domain. The parallelization of this step is similar to that of the diffusion step, while the tracking of the most advanced tip is performed between kernel calls on the CPU.

In this first implementation of the DNN model, we consider needles growing aligned along the finite difference grid, as illustrated for a needle growing in the $x$ direction in Fig. 3 . The restriction of a growth direction fixed to the finite difference grid is a convenience choice for the first implementation of the model, but not an intrinsic limitation of the model. The assumption of thick parabolic needle tips actually makes it easier to develop implementations of the DNN model with tilted grains (see e.g. Ref. [53]).

In the current thick needle formulation, interfaces are represented as steps, as in Fig. 3. Thus, we directly set $u=u_{i}$ on every point within the needle thickness, i.e. the green shaded zone in Fig. 3 within the analytical parabolic shape in blue dashed line. Even though this simple implementation provides an approximate description of the tip shape, e.g. the green solid profile inside the blue dashed parabola in Fig. 3, we found this simple description to be accurate enough and yield reasonably accurate results, as discussed in later sections.

Like the J-integral implementation presented in Ref. [36], the integration contour (or surface in 3D) $\Gamma$ is chosen to intersect the center of grid elements, as shown in Fig. 3. The first order derivatives for the flux through $\Gamma$ are calculated similarly as in Ref. [36], and the integration of the surface (volume in 3D) term over $\Sigma$ in Eq. (29) or (33) uses standard centered finite difference terms for each point within $\Sigma$, i.e. within the orange shaded region in Fig. 3. Since a needle tip radius and hence its parabolic thickness evolves, the width of the integration box $\Gamma$ in the direction normal to the needle is also set to evolve together with the parabolic shape of the tip. Hence, the shape of the integration box is parametrized using three user-input dimensions $A, B$, and $C$, such that for a needle growing in the $x$ direction the integration box spans $A$ grid points behind $i_{t i p}, B$ grid points ahead of $i_{t i p}$, and is $C$ grid points wider than the needle at its intersection with $\Gamma$ in the direction(s) normal to the needle. This is illustrated for $A=3, B=2$, and $C=1$ in Fig. 3 . Note that, in the calculation of the flux intensity factor $\mathcal{F}$, the integration distance $a$ between the tip of the needle and its intersection with $\Gamma$ is thus $a=(A+r+1 / 2) \Delta x$, with $r \Delta x$ the location of the tip between $i_{t i p}$ and $i_{t i p}+1$ (see Fig. 3).

An additional simplification is required to prevent numerical instabilities in the context of growth com- 
petition among several branches. During such growth competition, some branches stop as their tip velocity $V \rightarrow 0$. Thus, keeping the constancy of $\rho^{2} V$, the tip radius of the needle $\rho$ would tend to infinity, thus leading to an imposed condition $u=u_{i}$ over an unrealistically large domain. A solution to prevent the resulting numerical instabilities is to bound the thickness of all needles to a given thickness $r_{\text {max }}$. For instance in the 2D illustration of Fig. 3 with $r_{\max } / \Delta x=3$, the interface location $y_{i}$ follows a parabola $x=x_{t}-\left(y_{i}-y_{t}\right)^{2} /(2 \rho)$ as long as $\left|y_{i}-y_{t}\right| \leq r_{\max }$, and is bounded to $\left|y_{i}-y_{t}\right|=r_{\max }$ further behind the tip. Similarly in $3 \mathrm{D}$, the interface has a paraboloid shape in the vicinity of the tip, and is bounded to a cylinder of diameter $r_{\max }$ further behind the tip. We show in the following section that as long as the bounding of the needle thickness does not occur too close to the integration box $\Gamma$, forcing $r_{\max }$ has a small effect on the quantitive prediction of the tip growth dynamics.

\section{Analytical validations}

\subsection{Steady-state growth in $2 D$}

We compare DNN predictions for the steady-state growth of an isolated dendrite to the exact Ivantsov solution $[52,3]$. For isothermal conditions, we let a single needle grow within an imposed supersaturation $\Omega$ and measure the value of the Péclet number $P \equiv \rho V /(2 D)$ once $\rho$ and $V$ reach constant values.

First in two dimensions, we test three different formulations: (i) the sharp-needle formulation using the contour integral, Eq. (16), labeled Rice integral in Fig. 4; (ii) the sharp-needle formulation using the flux integral, Eq. (25), labeled Flux integral; and (iii) the thick-needle formulation using the FIF from Eqs. (25) and (29), labeled Parabolic needle. For sharp needle formulations, we set $\Delta x=10 \rho_{s}$ and test values of $\Omega$ from 0.01 to 0.1 by steps of 0.01 , and from 0.1 to 0.2 by steps of 0.02 , within a $(x \times y)$ domain of $1024 \times 1504$ grid points. The integration contour for the Rice integral is a square of side $6 \Delta x$ centered on the needle tip. For the sharp needle formulation with flux integration following Eq. (25), we set $a \approx 3 \Delta x$ (i.e. $A=3$ and $B=C=0$ with Fig. 3 notations). For the parabolic needle formulation, we test $\Omega$ from 0.04 to 0.2 by steps of 0.02 , within a domain of $2048^{2}$ grid points for $\Omega<0.08$ and $1024^{2}$ grid points for $\Omega \geq 0.08$, with a grid spacing $\Delta x=\rho_{s}$. The integration contour for the estimation of $\mathcal{F}$ is a rectangle that spans $5 \rho_{s}$ behind the tip and $2 \rho_{s}$ ahead of the tip in the $x$ direction, and in the $y$ direction is one $\rho_{s}$ wider than the needle at $5 \rho_{s}$ behind its tip - i.e. $A=5, B=2$ and $C=1$ following notations of Fig. 3. All boundaries are set to no-flux symmetry conditions. The needle is aligned along $(y=0)$ with its tip located at $1 / 5$ of the simulation domain in $x$.

Fig. 4 shows the DNN model predictions of steady-state growth conditions compared to the Ivantsov solution, Eq. (56), and its sharp-needle approximation, Eq. (60). For $\Omega<0.1$, all three formulations provide a reasonable estimation of the flux intensity factor $\mathcal{F}$, and hence of the steady-state of a growing needle. At higher $\Omega$, the Rice integral formulation, Eq. (16), exhibits a deviation from the sharp needle solution 
$\Omega \approx \sqrt{\pi P}(\times)$. Since the contour integral, Eq. (16), is based on the assumption of a Laplacian field around the tip, and does not include the velocity correction linked to the moving frame as in Eq. (29), this formulation does not remain accurate with a fixed size of the integration contour $\Gamma$, while the diffusion length gets smaller at higher $\Omega$. In contrast, the direct integration of the flux along a sharp needle following Eq. (25) yields predictions that follow $\Omega \approx \sqrt{\pi P}$ up to higher supersaturations $(+$ ). Furthermore, using a flux integration by Eqs. (25) and (29) and a parabolic needle, the thick-needle formulation closely predicts the exact Ivantsov solution (o). This illustrates two corrections compared to the sharp-needle model from Ref. [36]: (i) the solute field around the needle is affected by the shape and thickness of the needle (deviation between $\circ$ and + ), and (ii) the integrated flux accounts for the Laplacian field in a moving frame, through the velocity-dependent surface integral in Eq. (29) (deviation from + to $\times$ ).

\subsection{Steady-state growth in $3 D$}

We run similar simulations of the steady growth of one needle in three dimensions, in order to test the dependence of the results upon the finite difference grid spacing $\Delta x$. Hence, we compare in Fig. 5a the results with the $3 \mathrm{D}$ Ivantsov solution $\operatorname{Iv}_{3 \mathrm{D}}(P)$, i.e. Eq. (57). The Ivantsov relation in $2 \mathrm{D}, \operatorname{Iv}_{2 \mathrm{D}}(P)$, i.e. Eq. (56), also appears in Fig. 5a for comparison.

The significant difference between the $2 \mathrm{D}$ and $3 \mathrm{D}$ relations for $\Omega_{s}(P)$ has an important implication in terms of appropriate computational parameters. Since the DNN approach is built on the assumption that $\rho \ll D / V$, the finite difference grid spacing, typically of the same order as the tip radius, must also be small enough compared to the steady-state diffusion length $l_{D}=D / V_{s}$. As seen in Fig. 5a, in 2D, the ratio $\rho /(D / V) \sim P$ following $\operatorname{Iv}_{2 \mathrm{D}}(P)$ is comparatively small at any given $\Omega$, making the condition $\Delta x \approx \rho_{s} \ll l_{D}$ easier to fulfill. In order to reach quantitative predictions in $3 \mathrm{D}$, the choice of grid spacing $\Delta x \sim \rho_{s}$ is more severely bounded by $\Delta x \ll l_{D}$ than in $2 \mathrm{D}$. While this is not an extremely stringent condition, we for instance cannot afford to use $\Delta x$ as high as $10 \rho_{s}$ for any supersaturation as we can in $2 \mathrm{D}$.

The difference between solute redistribution dynamics in 2D and 3D also yields significant differences in terms of the time needed to reach a steady growth regime, as discussed later in the context of directional solidification experiments in Section 6.2.

First, we test different values of $l_{D} / \Delta x$, namely $40,20,15,10$, and 5 , for solute supersaturations $\Omega=0.001,0.002,0.005,0.01,0.02,0.05,0.1,0.2$, and 0.25 . For each $\Omega$, we simulate the steady growth of a single needle and measure the resulting tip Péclet number $P$, within a cubic domain of side $15 l_{D}$. The single needle is aligned along the $(y, z)=(0,0)$ line, with its tip fixed at $x=5 l_{D}$. The integration box $\Gamma$ for the calculation of $\mathcal{F}$ is with $A=B=2$ and $C=1$ following Fig. 3 notations. We do not bound the thickness of the needle, letting $r_{\max }=\infty$. Simulated times are at least $50 D / V_{s}^{2}$ to ensure that the steadystate is reached. The results, in Fig. $5 \mathrm{a}$, show that the predictions remain accurate as long as $l_{D} / \Delta x \geq 10$. Fig. 5b-d also illustrates that steady-state composition profiles remain close for $l_{D} / \Delta x \geq 10$. For a larger 
$\Delta x$, the steady-state growth velocity starts exhibiting important oscillations as the tip grows between two successive grid points. Hence, values of $P$ reported in Fig. 5a correspond to time averaged values of the velocity in the steady-state. For $\Delta x / l_{D}=5$, the Péclet number starts deviating from the well-converged solution, particularly showing strong oscillations at low supersaturation, and the composition profile also starts to differ noticeably in Fig. 5e compared to finer grids in Fig. 5b-d. In conclusion, keeping $\Delta x$ about an order of magnitude smaller than $l_{D}$ yields a reasonable accuracy on steady-state conditions. Moreover, while the converged predictions of the model follow the analytical Ivantsov solution, the current implementation appears to slightly underestimate $P$ at $\Omega \geq 0.01$ and to slightly overestimate $P$ at lower $\Omega$.

Next, we test the effect of bounding the thickness of a needle to a given $r_{\max }$, as presented in the last paragraph of Section 3.2 (see Fig. 3). While the simulations reported in Fig. 5 were achieved without restricting the thickness of the needle, i.e. $r_{\max }=\infty$, we performed similar simulations for $\Omega=0.02,0.05$, and 0.1 , with $l_{D} / \Delta x=40$ using different values of the maximum needle radius $r_{\max } / \rho_{s}=1,1.5,2,2.5,3,4$, $5,6,8,10,15$, and 20 . The integration box $\Gamma$ is set to $A=3, B=C=2$. Additionally, in order to estimate the effect of truncating the needle thickness for coarser finite difference meshes, we also carry out similar simulations for $\Omega=0.05$ and $l_{D} / \Delta x=10$. Figure 6 a shows the predicted steady-state Péclet number $P$ as a function of the truncating radius $r_{\max } / \rho_{s}$, and Fig. 6b-f illustrate the needle shape and composition profile in the vicinity of the tip for $\Omega=0.05, l_{D} / \Delta x=40$, and different values of $r_{\max }$.

As shown previously (Fig. 5a), for $r_{\max } \rightarrow \infty$, the current implementation slightly underestimates $P$ for $\Omega \geq 0.01$ (by about $10 \%$ ). When reducing $r_{\max }$, the absence of thickness of the needle tail at $u=0$ shrinks the the solute profile towards the needle, which in turn leads to higher gradients in the vicinity of the tip, as shown in Fig. 6b-f. Thus, the incoming flux at the tip, the resulting $\mathcal{F}$, the steady-state velocity, and ultimately the Péclet number $P$ increase when $r_{\max }$ decreases up to about $1.5 \rho_{s}$ for $\Omega=0.1$. For lower values of $r_{\max }$, the truncating of the needle thickness occurs closer to the tip, i.e. within the integration domain $\Gamma$. The overlapping of the truncated domain with the integration box $\Gamma$ induces a decrease of $\mathcal{F}$ and of the steady $P$ as $r_{\max }$ decreases. Note that, for lower $\Omega$ values, $l_{D}$ is higher, then $\Delta x / \rho_{s}$ is also higher for a given $l_{D} / \Delta x$, and the maximum of $P$ in Fig. 6 a occurs at a higher $r_{\max } / \rho_{s}$.

This general behavior is common to all three supersaturations and also holds for a coarser mesh with $l_{D} / \Delta x=10$. In conclusion, the variation in $\mathcal{F}$ with $r_{\max }$ is limited as long as $r_{\max }$ is chosen larger or equal to a few $\Delta x$ such that truncation of the needle thickness does not occur within the integration box $\Gamma$.

\subsection{Early transient growth in $3 D$}

We have shown in Ref. [36] that, for $\Omega \ll 1$ the sharp needle model in $2 \mathrm{D}$ reproduces the analytical solution for an equiaxed four-branch crystal with the branch length following the power law $L \sim t^{3 / 5}[54]$. In $3 \mathrm{D}$, dimensional analysis and phase field calculations have shown that the isothermal growth of an equivalent idealized six-branch crystal yields $L \sim t^{2 / 3}$ [49]. Thus, we use the 3D DNN model to calculate 
the isothermal growth of an equiaxed six-branch cross for $P \approx 0.00125$, i.e. $\Omega \approx 0.00634$.

Figure 7 a shows that the DNN model predicts this $L \sim t^{2 / 3}$ behavior over three orders of magnitude in time. Due to the complexity of simulating three orders of magnitude in time with a single calculation, Fig. $7 \mathrm{a}$ is build by continuity from three simulations with $\Delta x / \rho_{s}=0.05$ (for $t V_{s} / \rho_{s} \leq 2$ ), 0.1 (for $2 \leq t V_{s} / \rho_{s} \leq 10$ ), and 0.5 (for $t V_{s} / \rho_{s} \geq 10$ ). The simulations were achieved on a domain of $904^{3}$ grid points, with a integration surface $\Gamma$ with $A=3, B=C=2$ (see Fig. 3), and no bounding of the needle thickness (i.e. $r_{\max }=\infty$ ).

These results show that, while a good prediction of steady state growth conditions can be achieved using a numerical grid size of the same order or slightly higher than a dendrite tip radius (see Sec. 4.1-4.2), DNN simulations also yield accurate predictions on transient stage at a much smaller time scale if spatial discretization is also made finer.

\section{Growth of sidebranches}

\subsection{Theory and experimental measurements}

The incorporation of anisotropic surface tension has been shown theoretically [48, 58] and by phasefield simulations [56] to cause the steady-state dendrite shape to deviate from Ivantsov's paraboloid of revolution [52]. With increasing distance behind the tip, the shape becomes progressively nonaxisymmetric and develops four fins with a shape predicted to follow the scaling law $\bar{l}_{2} \sim \bar{x}^{0.6} \quad[55]$ where $\bar{x} \equiv\left|x-x_{t}\right| / \rho$ and $\bar{l}_{2}=l_{2} / \rho$ where $l_{2}$ is the width of the fin measured from the central dendrite growth axis. This scaling law was derived based on the assumption that the cross-section of a 3D steady-state dendrite evolves with distance from the tip similarly to a $2 \mathrm{D}$ equiaxed grain in time and has been found to be in good agreement with both experiments [57] and phase-field simulations [56]. This scaling law is expected to lose validity once sidebranches emerge from the fins and grow competitively behind the tip, screening each other via the solutal diffusion field, until a branch ultimately becomes long enough to grow as a free dendrite. Experimental measurements on succinonitrile-acetone alloys by Beckermann and co-workers have shown that the envelope of actively growing secondary branches, i.e. the length $\bar{l}_{2}$ of those sidebranches follows a power law $\bar{l}_{2} \approx 0.84 \bar{x}^{0.85}$ until about 200 tip radii behind the primary tip, which was found to be independent of undercooling or alloy concentration in a low Péclet number regime (i.e. for about $\Omega \approx 0.041$ to 0.24 in Fig. 14b of Ref. [39]). This scaling law has remained challenging to test computationally owing to the daunting task of simulating the evolution of secondary branches in 3D in a low Péclet number regime on a scale comparable to the diffusion length. While it can be reproduced using a grain envelope tracking model [13], the latter does not reproduce the dynamics branch competition with elimination and overgrowth of secondary branches. This task is also beyond the scope of phase-field modeling, but well within the scope of the 3D DNN approach that we use here to characterize the scaling behavior of the competitive growth of well-developed sidebranches in 3D. 


\subsection{Comparison to DNN results}

To characterize the sidebranch dynamics, we perform a DNN simulation of isothermal solidification for $\Omega=0.1$, with $\Delta x=0.5 \rho_{s}$ (i.e. $l_{D} / \Delta x \approx 23$ ) on $800^{3}$ grid points over a time $2500 \rho_{s} / V_{s}$. The boundaries of the domain are symmetric (i.e. no-flux conditions). The initial needle is located along the line $(y, z)=(0,0)$. The simulation domain is shifted to keep the primary tip location at $60 \%$ of the domain in $x$ (i.e. $x_{t} \approx 240 \rho_{s}$ ). The thickness of the needles is bounded by $r_{\max }=4 \rho_{s}$. The integration box $\Gamma$ spans $2 \Delta x$ behind and ahead of the tip along $x$ and is one $\Delta x$ wider than the needle thickness at its intersection with $\Gamma$, i.e. with $A=B=2$, and $C=1$. Sidebranching events are set to occur at a distance behind the tip in the range of $5 \rho$ to $9 \rho$, i.e. $N=7$ and $\Delta N=4$ (see Section 2.6).

By the time $t=250 \rho_{s} / V_{s}$, the primary dendrite tip reaches a steady-state growth regime, marked with a full yellow circle on Fig. 5a. We allow the system to stabilize to a steady sidebranching regime, reached once the first few generations of secondary branches have left the domain due to the primary tip location being fixed at $x_{t} \approx 240 \rho_{s}$. At $t \geq 1000 \rho_{s} / V_{s}$, the primary tip has grown by more than twice its length in the simulation domain and the dendrite has reached a steady sidebranching regime, illustrated in the inset of Fig. 8 for $t V_{s} / \rho_{s} \approx 1700$.

We track the tip position of active secondary sidebranches in both $y$ and $z$ directions. Active sidebranches are defined as branches longer than other sidebranches that have their root closer to the primary tip. We output the active tip positions $\bar{l}_{2}(\bar{x})$ for $1000 \leq t V_{s} / \rho_{s} \leq 2500$ with time steps of 50, and fit the resulting cloud of points to a power law for $\bar{x} \leq 100$. The corresponding points from $t V_{s} / \rho_{s}=1000$ to 2500 with step of 250 appear as symbols in Fig. 8 , together with the fitted power law $\bar{l}_{2}=0.63 \bar{x}^{0.87}$ (solid line). Up to $\bar{x} \leq 100$, the simulated dendrite exhibits a good agreement with the experimentally assessed power law [39] (dashed line) with a very close exponent and a slightly lower prefactor. After $\bar{x}=100$, a few secondary sidebranches deviate from this power law as they start penetrating the diffusion field and transitioning toward a free dendrite growth regime.

\section{Application to experiments}

\subsection{Simulations}

We apply the 3D DNN model to simulate the three-dimensional directional solidification experiment of an aluminum-silicon alloy in reduced gravity conditions, achieved in the framework of the CETSOL project $[45,46,47]$. In this experiment, cylindrical ingots of diameter $7.8 \mathrm{~mm}$ are solidified over a length of several tens of centimeters onboard the International Space Station, hence yielding a purely diffusive transport regime and homogeneous growth conditions. The specific experiment simulated here was performed on an Al-7wt\%Si alloy with a temperature gradient $G=9 \mathrm{~K} / \mathrm{cm}$ and a pulling velocity $V_{p}=10 \mu \mathrm{m} / \mathrm{s}$ (i.e. Stage I for FM3 and FM4 in Refs [46, 47]). 
The considered alloy parameters are summarized in Table 1 . The value of $\sigma=0.058$ was chosen to match the atomistic simulations prediction of $\epsilon_{4}=0.012$ for aluminum [60] and linear solvability theory in 3D [43]. The simulation domain is made of $736 \times 576 \times 288$ grid points, with $\Delta x=2 \rho_{s}$ and $\rho_{s} \approx 7 \mu \mathrm{m}$, i.e. a domain of about $10.3 \times 8.0 \times 4.0 \mathrm{~mm}^{3}$, over an experimental time of 90 minutes. Within that domain, we impose no-flux, i.e. symmetric, boundary conditions along all boundaries and along the side of a cylinder of diameter $7.8 \mathrm{~mm}$ with its axis within the $(z=0)$ plane and centered in the $y$ direction, hence simulating one half of the cylindrical mold. The cylindrical shape of the mold is approximated with steps along $y$ or $z$, hence combining no-flux conditions solely on $y$ and solely on $z$ (see e.g. schematics in the Supplementary Material of Ref. [61]). The integration box $\Gamma$ for the calculation of $\mathcal{F}(t)$ is set to $A=B=3$, and $C=1$ (see Fig. 3). The thickness of the needles is bounded to $r_{\max }=3 \rho_{s}$. The domain is shifted to keep the primary dendrite tips at $x \approx 8.32 \mathrm{~mm}$. Branching events are set to occur every 10 to $20 \rho$, i.e. with $N=15$ and $\Delta N=10$. The initial network is a grid of 390 needles oriented in the $x$ direction and evenly spaced in $y$ and $z$ with steps of $18 \Delta x$. The needle tips are initially aligned at the liquidus temperature, so as to mimic a planar front, with a small random fluctuation of amplitude $0.001 \rho_{s}$ on their initial length in order to disturb the initial symmetry of the problem [36].

\subsection{Results}

The resulting simulation is widely multiscale, with a steady-state capillarity length $d_{0}^{*} \approx 4.75 \mathrm{~nm}$, a steady primary tip radius $\rho_{s} \approx 7.0 \mu \mathrm{m}$, a diffusion length $l_{D}=300 \mu \mathrm{m}$, and a thermal length $l_{T} \approx 33.8 \mathrm{~cm}$. The simulation runs in about 28 hours on a single Nvidia GTX680 ${ }^{\circledR}$ GPU.

The results of the simulation are illustrated in Fig. 9. The planar front reaches a maximum dimensionless undercooling of about 0.016 , i.e. a tip supersaturation $\Omega \approx 0.11$, at $t \approx 630$ s before destabilizing, accelerating, and finally stabilizing at an undercooling of 0.0072 , i.e. $\Omega \approx 0.053$, with a steady Péclet number of approximately 0.0116, marked in Fig. 5a with an open yellow circle. The total number of needles in the simulation peaks slightly above 25000 as the interface accelerates shortly after the planar front destabilization, then stabilizing around 12000 needles in the steady-state. The maximum memory (RAM) required during the simulation remains below $2 \mathrm{~GB}$, which makes the simulation accessible to almost any commercial GPU released in the past few years. The snapshots in Fig. 9 show the initial array of needles at $t=0$, the dendritic array shortly after the planar front destabilization at $t=18 \mathrm{~min}$, and the subsequent elimination/coarsening of the array at $t=30 \mathrm{~min}$ until the steady-state shown at $t=90 \mathrm{~min}$.

We compare these results with the corresponding 2D simulation from Ref. [36]. Fig. 10 shows the time evolution of the supersaturation of the most advanced primary tip in the dendritic array in $2 \mathrm{D}$ (dashed) and 3D (solid) simulations. The longer transient in 2D is primarily due to the difference in solute redistribution and to the resulting difference in $\Omega_{s}$ between $2 \mathrm{D}$ and 3D, as illustrated with the two steady-states marked with yellow symbols in Fig. 5a. The smaller effect of the tip selection parameter $\sigma(0.083$ in $2 \mathrm{D}$ and 0.058 in 
3D) also appears in Fig. $5 \mathrm{a}$ as the difference in the steady Péclet number that scales as $P \sim 1 / \sqrt{\sigma}$, if other parameters remain the same. The significant difference in time scale to reach a steady growth highlights the importance of three-dimensional simulations to predict reasonable transient dynamics. The difference in solute profiles ahead of the dendritic front may also yield significant differences in selected primary spacings, as shown elsewhere for a directionally solidified $\mathrm{Al}-\mathrm{Cu}$ alloy compared to x-ray radiography imaging [62].

\subsection{Comparison to experiments}

We compare the resulting steady-state microstructures to experimental microstructures observed in a CETSOL experiment in Fig. 11. A count of primary dendritic trunks within the cross section (a,b) and across the longitudinal section of the sample $(\mathrm{c}, \mathrm{d})$ shows a good agreement between simulations and experiments. Note that the diagonal lines through Fig. 11d are geometrical artifacts due to the cutting and polishing plane not exactly parallel to the dendrite growth direction, while the latter is actually only a few degrees tilted with respect to the vertical temperature gradient direction.

This first direct comparison of simulations with experiments on a crucial microstructural feature such as primary dendritic spacing is a very encouraging example of the potential of the DNN approach in predicting quantitatively fully three-dimensional microstructures at the scale of cubic centimeters and hours with reasonably sized computations.

\section{Summary and outlook}

We presented a three-dimensional formulation of the Dendritic Needle Network (DNN) model for dendritic growth for isothermal and directional solidification. We have shown that this approach describes quantitatively analytical solutions for both steady-state and early stage growth regimes, using a spatial discretization of the same order or larger than a dendrite tip radius. The model also reproduces quantitatively the experimentally observed scaling law relating the growth of the length of active secondary branches to the distance behind the tip, which determines the envelope shape of the dendritic grain. We applied DNN simulations to three-dimensional directional solidification of an Al-7wt\%Si alloy in a purely diffusive regime in a microgravity experiment performed in the framework of the CETSOL project. These results, as well as first applications of the model to other $\mathrm{Al}-\mathrm{Si}$ and $\mathrm{Al}-\mathrm{Cu}$ alloys in thin sample experiments published elsewhere [61, 62], show a good agreement between primary spacings experimentally measured and those predicted by the DNN model.

The present modeling approach reliably bridges the length-scale gap between detailed solid-liquid interface pattern formation models, e.g. phase-field, and macroscopic average models of grain growth, e.g. cellular automata. The ability to model and explore both intragrain and intergrain dynamical phenomena in complex dendritic microstructures should pave the way for a deeper understanding of microstructure 
selection mechanisms, such as for dendrite arm spacings, grain texture, and casting defects, crucial to the properties of industrial materials.

The outlooks of this work are manyfold. The new formulation involving thicker parabolic needles instead of sharp needles makes it easier to build a numerical implementation where the branches are not aligned along the computational grid. This opens the way to polycrystalline implementations of the model that account for different grain orientations, as well as modeling the growth of crystals with hexagonal symmetry. Additionally, in order to model solidification under realistic casting conditions, the DNN approach needs to be extended to treat thermo-solutal transport, multicomponent alloys, and fluid flow. Furthermore, even though the DNN model is not designed to model coarsening and latter stage solidification at high solid fraction, introducing a simplified description of solidification in the mushy zone would be a valuable addition to the model in order to predict fully solid dendritic microstructures.

We expect the DNN model and its extensions to yield deeper insight into microstructure selection, exploring for instance the mechanisms of polycrystalline grain growth competition [35], the influence of convective transport at the scale of extended dendritic arrays [63], or the columnar-to-equiaxed transition [45, $46,47]$.

\section{Acknowledgements}

The development of the model was supported by the National Aeronautics and Space Administration through grants NNX11AC09G and NNX16AB54G. For the writing of this article and numerical applications of the model, DT gratefully acknowledges the support of the U.S. Department of Energy LANL/LDRD Program through a Director's Postdoctoral Fellowship.

\section{References}

[1] M.C. Flemings, Solidification processing, McGraw-Hill, New York, 1974.

[2] R. Trivedi, W. Kurz, Dendritic growth, Int. Mater. Rev. 39 (1994) 49-74.

[3] W. Kurz, D.J. Fisher, Fundamentals of solidification, 3rd ed., Trans Tech Pub, Aedermannsdorf, 1992.

[4] J.A. Dantzig, M. Rappaz, Solidification, EPFL Press, Switzerland, 2009.

[5] J. Ni, C. Beckermann, A volume-averaged two-phase model for transport phenomena during solidification, Metall. Mater. Trans. B 22 (1991) 349-361.

[6] C.Y. Wang, C. Beckermann, Equiaxed dendritic solidification with convection: Part I. Multiscale/multiphase modeling, Metall. Mater. Trans. A 27 (1996) 2754-2764.

[7] M. Wu, A. Ludwig, Modeling equiaxed solidification with melt convection and grain sedimentation - I: Model description, Acta Mater. 57 (2009) 5621-5631.

[8] M. Wu, A. Ludwig, Modeling equiaxed solidification with melt convection and grain sedimentation - II. Model verification, Acta Mater. 57 (2009) 5632-5644.

[9] I. Steinbach, C. Beckermann, B. Kauerauf, Q. Li, J. Guo, Three-dimensional modeling of equiaxed dendritic growth on a mesoscopic scale, Acta Mater. 47 (1999) 971-982. 
[10] I. Steinbach, H-J. Diepers, C. Beckermann, Transient growth and interaction of equiaxed dendrites, J. Cryst. Growth 275 (2005) 624-638.

[11] A. Kharicha, M. Stefan-Kharicha, A. Ludwig, M. Wu, A scale adaptive dendritic envelope model of solidification at mesoscopic scales, IOP Conf. Ser.: Mater. Sci. Eng. 84 (2015) 012032.

[12] M. Založnik, A. Viardin, Y. Souhar, H. Combeau, M. Apel, Mesoscopic modeling of columnar solidification and comparisons with phase-field simulations, IOP Conf. Ser.: Mater. Sci. Eng. 84 (2015) 012074

[13] Y. Souhar, V.F. De Felice, C. Beckermann, H. Combeau, M. Založnik, Three-dimensional mesoscopic modeling of equiaxed dendritic solidification of a binary alloy, Comp. Mater. Sci. 112 (2016) 304-317.

[14] M. Rappaz, Ch.-A. Gandin, Probabilistic modelling of microstructure formation in solidification processes, Acta Metall. Mater. 41 (1993) 345-360.

[15] Ch.-A. Gandin, M. Rappaz, A coupled finite element-cellular automaton model for the prediction of dendritic grain structures in solidification processes, Acta Metall. Mater. 42 (1994) 2233-2246.

[16] Ch.-A. Gandin, Modeling of solidification: Grain structures and segregations in metallic alloys, C. R. Physique 11 (2010) 216-225.

[17] W. Wang, P.D. Lee, M. McLean, A model of solidification microstructures in nickel-based superalloys: predicting primary dendrite spacing selection, Acta Mater. 51 (2003) 2971-2987.

[18] M. Eshraghi, S.D. Felicelli, B. Jelinek, Three dimensional simulation of solutal dendrite growth using lattice Boltzmann and cellular automaton methods, J. Cryst. Growth 354 (2012) 129-134.

[19] M. Eshraghi, B. Jelinek, S.D. Felicelli, Large-Scale Three-Dimensional Simulation of Dendritic Solidification Using Lattice Boltzmann Method, JOM 67 (2015) 1786-1792.

[20] J.D. Miller, L. Yuan, P.D. Lee, T.M. Pollock, Simulation of diffusion-limited lateral growth of dendrites during solidification via liquid metal cooling, Acta Materialia 69 (2014) 47-59.

[21] Ch.-A. Gandin, T. Carozzani, H. Digonnet, S. Chen, G. Guillemot, Direct Modeling of Structures and Segregations Up to Industrial Casting Scales, JOM 65 (2013) 1122-1130.

[22] W.J. Boettinger, J.A. Warren, C. Beckermann, A. Karma, Phase-field simulation of solidification, Annu. Rev. Mater. Res. $32(2002) 163$.

[23] A. Karma, W.J. Rappel,Quantitative phase-field modeling of dendritic growth in two and three dimensions, Phys. Rev. E 57 (1998) 4323.

[24] B. Echebarria, R. Folch, A. Karma, M. Plapp, Quantitative phase-field model of alloy solidification, Phys. Rev. E 70 (2004) 061604 .

[25] A. Karma, Phase-Field Formulation for Quantitative Modeling of Alloy Solidification, Phys. Rev. Lett. 87 (2001) 115701.

[26] E.A. Brener, G. Boussinot, Kinetic cross coupling between nonconserved and conserved fields in phase field models, Phys. Rev. E 86 (2012) 060601.

[27] G. Boussinot, E.A. Brener, Achieving realistic interface kinetics in phase-field models with a diffusional contrast, Phys. Rev. E 89 (2014) 060402.

[28] N. Provatas, N. Goldenfeld, J. Dantzig, Efficient Computation of Dendritic Microstructures Using Adaptive Mesh Refinement, Phys. Rev. Lett. 80 (1998) 3308.

[29] N. Provatas, M. Greenwood, B. Athreya, N. Goldenfeld, J. Dantzig, Multiscale modeling of solidification: phase-field methods to adaptive mesh refinement, Int. J. Mod. Phys. B 19 (2005) 4525-4565.

[30] W.L. George, J.A. Warren, A parallel 3D dendritic growth simulator using the phase-field method, J. Comput. Phys. B 177 (2002) 264-283.

[31] T. Shimokawabe, T. Takaki, T. Endo, A. Yamanaka, N. Maruyama, T. Aoki, A. Nukada, S. Matsuoka, Peta-scale phasefield simulation for dendritic solidification on the TSUBAME 2.0 supercomputer, in: 2011 International Conference for 
High Performance Computing, Networking, Storage and Analysis (SC), IEEE, 2011, pp. 1-11.

[32] N. Bergeon, D. Tourret, L. Chen, J.-M. Debierre, R. Guérin, A. Ramirez, B. Billia, A. Karma, R. Trivedi, Spatiotemporal Dynamics of Oscillatory Cellular Patterns in Three-Dimensional Directional Solidification, Phys. Rev. Lett. 110 (2013) 226102.

[33] D. Tourret, J.-M. Debierre, Y. Song, F.L. Mota, N. Bergeon, R. Guérin, R. Trivedi, B. Billia, A. Karma, Cellular pattern oscillations in three-dimensional directional solidification, Phys. Rev. E 92 (2015) 042401.

[34] T. Takaki, T. Shimokawabe, M. Ohno, A. Yamanaka, T. Aoki, Unexpected selection of growing dendrites by very-largescale phase-field simulation, J. Cryst. Growth 382 (2013) 21-25.

[35] D. Tourret, A. Karma, Growth competition of columnar dendritic grains: A phase-field study, Acta Mater. 82 (2015) 64-83.

[36] D. Tourret, A. Karma, Multiscale dendritic needle network model of alloy solidification, Acta Mater. 61 (2013) $6474-6491$.

[37] D. Tourret, A. Karma, Corrigendum to: "Multi-scale dendritic needle network model of alloy solidification" [Acta Mater. 61 (2013) 6474-6491], Acta Mater. 87 (2015): 411.

[38] Y. Couder, J. Maurer, R. González-Cinca, A. Hernández-Machado, Side-branch growth in two-dimensional dendrites. I. Experiments, Phys. Rev. E 71 (2005) 031602.

[39] A.J. Melendez, C. Beckermann, Measurements of dendrite tip growth and sidebranching in succinonitrile-acetone alloys, J. Cryst. Growth 340 (2012) 175-189.

[40] J.R. Rice, A path independent integral and the approximate analysis of strain concentration by notches and cracks, J. Appl. Mech. 35 (1968) 379-386.

[41] J.S. Langer, Lectures on the Theory of Pattern Formation, in: Chance and Matter (Les Houches, Session XLVI), J. Souletie, J. Vannimenus, R. Stora (Eds.), North-Holland, Amsterdam, 1987, pp. 629-711.

[42] J.S. Langer, Dendrites, Viscous Fingers, and the Theory of Pattern Formation, Science 243 (1989) 1150-1156.

[43] A. Barbieri, J.S. Langer, Predictions of dendritic growth rates in the linearized solvability theory, Phys. Rev. A 39 (1989) 5314.

[44] Y. Shibuta, M. Ohno, T. Takaki, Solidification in a Supercomputer: From Crystal Nuclei to Dendrite Assemblages, JOM 67 (2015) 1793-1804.

[45] G. Zimmermann, L. Sturz, B. Billia, N. Mangelinck-Noël, H. Nguyen Thi, Ch.-A. Gandin, D.J. Browne, W.U. Mirihanage, Investigation of columnar-to-equiaxed transition in solidification processing of AlSi alloys in microgravity - The CETSOL project, J. Phys.: Conf. Ser. 327 (2011) 012003.

[46] G. Zimmermann, L. Sturz, B. Billia, N. Mangelinck-Noël, D.R. Liu, H. Nguyen Thi, N. Bergeon, Ch.-A. Gandin, D.J. Browne, C. Beckermann, D. Tourret, A. Karma, Columnar-to-Equiaxed Transition in Solidification Processing of AlSi7 Alloys in Microgravity - The CETSOL Project, Mater. Sci. Forum 790 (2014) 12-21.

[47] D.R. Liu, Mangelinck-Noël N, Ch.-A. Gandin, G. Zimmermann, L. Sturz, H. Nguyen Thi, B. Billia, Structures in directionally solidified Al-7wt.\% Si alloys: Benchmark experiments under microgravity, Acta Mater. 64 (2014) 253-265.

[48] M. Ben Amar, E. Brener, Theory of Pattern Selection in Three-Dimensional Nonaxisymmetric Dendritic Growth, Phys. Rev. Lett. 71 (1993) 589.

[49] M. Plapp, A. Karma, Multiscale Random-Walk Algorithm for Simulating Interfacial Pattern Formation, Phys. Rev. Lett. $84(2000) 1740$.

[50] B. Derrida, V. Hakim, Needle models of Laplacian growth, Phys. Rev. A 45 (1992) 8759.

[51] M. Ben Amar, J.R. Rice, Exact results with the J-integral applied to free-boundary flows, J. Fluid Mech. 461 (2002) $321-341$.

[52] G.P. Ivantsov, Temperature field around a spherical, cylindrical, and needle-shaped crystal, growing in a pre-cooled melt, Dokl. Akad. Nauk. SSSR 58 (1947) 567-569. 
[53] P.A. Geslin, A. Karma, Numerical investigation of the columnar-to-equiaxed transition using a 2D needle network model, in: TMS 2016 Annual Meeting, Supplemental Proceedings, Symposium Frontiers in Solidification, 2016, pp. 29-34.

[54] R. Almgren, W.S. Dai, V. Hakim, Scaling Behavior in Anisotropic Hele-Shaw Flow, Phys. Rev. Lett. 71 (1993) 3461.

[55] E. Brener, Needle-crystal solution in three-dimensional dendritic growth, Phys. Rev. Lett. 71 (1993) 3653.

[56] A. Karma, Y.H. Lee, M. Plapp, Three-dimensional dendrite-tip morphology at low undercooling, Phys. Rev. E 61 (2000) 3996.

[57] U. Bisang, J.H. Bilgram, Shape of the Tip and the Formation of Sidebranches of Xenon Dendrites, Phys. Rev. Lett. 75 (1995) 3898.

[58] E. Brener, V.I. Melnikov, Velocity selection and instability spectrum in 3D dendritic growth, Sov. J. Exp. Th. Phys. 80 (1995) 341-345.

[59] Ch.-A. Gandin, From constrained to unconstrained growth during directional solidification, Acta Mater. 48 (2000) $2483-$ 2501.

[60] J.R. Morris, The Anisotropic Free Energy of the Solid-Liquid Phase Boundary in Al, Phys. Rev. B 66 (2002) 144104.

[61] D. Tourret, A.J. Clarke, S.D. Imhoff, P.J. Gibbs, J.W. Gibbs, A. Karma, Three-dimensional multiscale modeling of dendritic spacing selection during Al-Si directional solidification, JOM 67 (2015) 1776-1785.

[62] D. Tourret, A. Karma, A.J. Clarke, P.J. Gibbs, S.D. Imhoff, Three-dimensional Dendritic Needle Network model with application to Al-Cu directional solidification experiments, IOP Conf. Ser.: Mater. Sci. Eng. 84 (2015) 012082.

[63] I. Steinbach, Pattern formation in constrained dendritic growth with solutal buoyancy, Acta Mater. 57 (2009) $2640-2645$. 


\section{List of Figures}

1 The Dendritic Needle Network model represents a dendritic grain, like the ammoniumbromide crystal from Ref. [38] in (a), as a hierarchical network of needle-like branches interacting through the long range diffusion field, as illustrated in (b). The instantaneous tip radius $\rho(t)$ and velocity $V(t)$ of each branch is established by combining two conditions at distinct length scales: (c) a solute conservation condition at an intermediate scale larger than the tip radius $\rho$, but smaller than the diffusion length $D / V$ (indicated with the $\gg \rho$ and $\ll D / V$ scale bar labels, respectively), and (d) a solvability condition at the scale of the tip

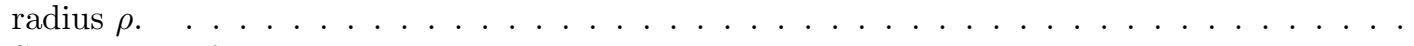

2 Schematics of a dendritic tip at an intermediate scale between the tip radius $\rho$ and the much larger diffusion length $D / V$ (indicated with the $\gg \rho$ and $\ll D / V$ scale bar labels, respectively) for (a) a sharp and (b) a parabolic needle crystal. . . . . . . . . . . . . . . .

3 Illustration of a needle tip region. The integration contour (surface in $3 \mathrm{D}$ ) $\Gamma$ and its inner surface (volume in 3D) $\Sigma$ spans $A$ grid points behind the tip location $\left(i_{\text {tip }}, j_{\text {tip }}\right), B$ grid points ahead of the tip, and is $C$ grid points wider than the needle at its intersection with $\Gamma$ - here illustrated for $A=3, B=2$, and $C=1$. The needle width (radius in 3D) is bounded to a maximum $r_{\max }-$ here equal to $3 \Delta x \ldots \ldots \ldots \ldots \ldots$

4 Steady-state Péclet number for different solute supersaturations $\Omega$ in two dimensions, compared to the exact Ivantsov solution, and its sharp-needle approximation $\Omega \approx \sqrt{\pi P}$, for three different DNN model formulations for the estimation of the flux intensity factor $\mathcal{F}:(\times)$ using the Rice integral, Eq. (16), as in Ref. [36]; (+) using the flux integral, Eq. (25), for a sharp needle; and (o) using a thick parabolic needle tip with Eqs. (25) and (29). . . . . . . . . . .

5 Steady-state supersaturation $\Omega$ versus Péclet number $P$ from DNN simulations in three dimensions with $r_{\max }=\infty$ for $l_{D} / \Delta x=40,20,15,10$, and 5 (a). For $\Omega=0.05$, panels (b) through (e) show isovalues of the composition field $u$ in the $(z=0)$ plane with steps of 0.005 and the interface position, i.e. $u=0$, in thicker blue line, for $l_{D} / \Delta x=40$ (b), 15 (c), 10 (d), and 5 (e). Yellow symbols in (a) show the steady-state for a simulation with sidebranches (Fig. 8) and directional solidification simulations (Figs 9-11) . . . . . . . . . . . .

$6 \quad$ Predicted steady-state Péclet number $P$ as a function of the needle thickness bounding radius $r_{\max }(\mathrm{a})$, and corresponding composition profiles in the $(z=0)$ plane, with contour steps of 0.005 , for $\Omega=0.05, l_{D} / \Delta x=40$ and $r_{\max } / \rho_{s}=20(\mathrm{~b}), 8(\mathrm{c}), 5$ (d), 3 (e), and 1 (f). . . . .

7 Early growth of a six-branch equiaxed crystal predicted by 3D DNN simulations for $\Omega \approx$ 0.00634 , i.e. $P \approx 0.00125$. In panel (a) the evolution of the branches length $L$ is compared in a log-log plot to a line of slope $2 / 3$, corresponding to the analytical law $L \sim t^{2 / 3}$ [49]. The plot is built by continuity from simulations with $\Delta x=0.05 \rho_{s}$ (for $t V_{s} / \rho_{s} \leq 2$ ), $\Delta x=0.1 \rho_{s}$ (for $2 \leq t V_{s} / \rho_{s} \leq 10$ ), and $\Delta x=0.5 \rho_{s}$ (for $t V_{s} / \rho_{s} \geq 10$ ). Panels (b) through (g) show snapshots of the isovalues of $u$ with steps of 0.0005 , at times marked with circles in (a) . . . . . . . .

8 Sidebranches growth competition and resulting dendrite envelope for $\Omega=0.1$. Symbols mark the location of the active secondary sidebranches in the $y$ (red circles) and $z$ (blue diamonds) directions for $t V_{s} / \rho_{s}=1000$ to 2500 (shown with time steps of $250 \rho_{s} / V_{s}$ ). The power law fit of these locations (fitted with time steps of $50 \rho_{s} / V_{s}$ ) yields $\bar{l}_{2}=0.63 \bar{x}^{0.87}$ (solid line), as compared to measurements by Melendez and Beckermann [39] (dashed line). The inset shows the dendrite at $t V_{s} / \rho_{s}=1700$ with contour lines for $u=0.005$ to 0.095 with steps of 0.005

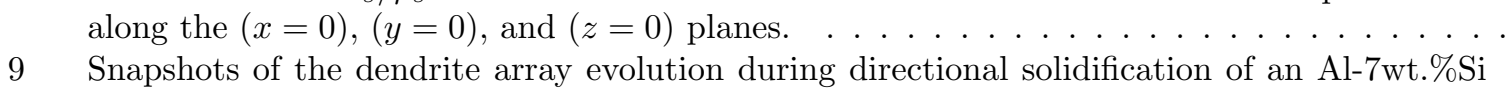
alloy at $V=10 \mu \mathrm{m} / \mathrm{s}$ and $G=9 \mathrm{~K} / \mathrm{cm}$ in a cylinder of diameter $7.8 \mathrm{~mm}$. Contour lines in (a) and (b) show isovalues of the $u$ field along the side of the cylindrical mold, with steps of 0.01 . The needle network is shown in (c) for $z \leq 1121 \mu \mathrm{m}$ with isovalues of the $u$ field along the $(z=1121 \mu \mathrm{m})$ plane, and in (d) for $x \geq 4205 \mu \mathrm{m}$ with contour lines on the $(z=4205 \mu \mathrm{m})$ plane. [Both planes are drawn on the rightmost images in (c) and (d)] . . . . . . . . . . 3 
10 Transient evolution of dendritic front supersaturation for a 2D simulation from Ref. [36] (dashed line) and the present 3D simulation in a cylinder (solid). The slight difference in initial slope is due to the $2 \mathrm{D}$ simulation using $G=10 \mathrm{~K} / \mathrm{cm}$ (while here $G=9 \mathrm{~K} / \mathrm{cm}$ ).

11 Microstructure cross-sections $(\mathrm{a}, \mathrm{b})$ and longitudinal sections $(\mathrm{c}, \mathrm{d})$ from a microgravity directional experiment of an Al-7wt.\%Si alloy at $V=10 \mu \mathrm{m} / \mathrm{s}$ and $G=9 \mathrm{~K} / \mathrm{cm}$ in the CETSOL project (b,d), adapted from Ref [46], and from a 3D DNN simulation (a,c). Each panel shows

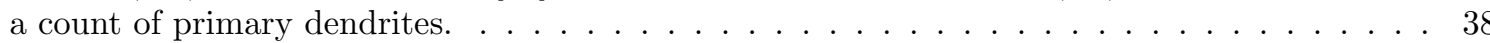




\section{Figures}

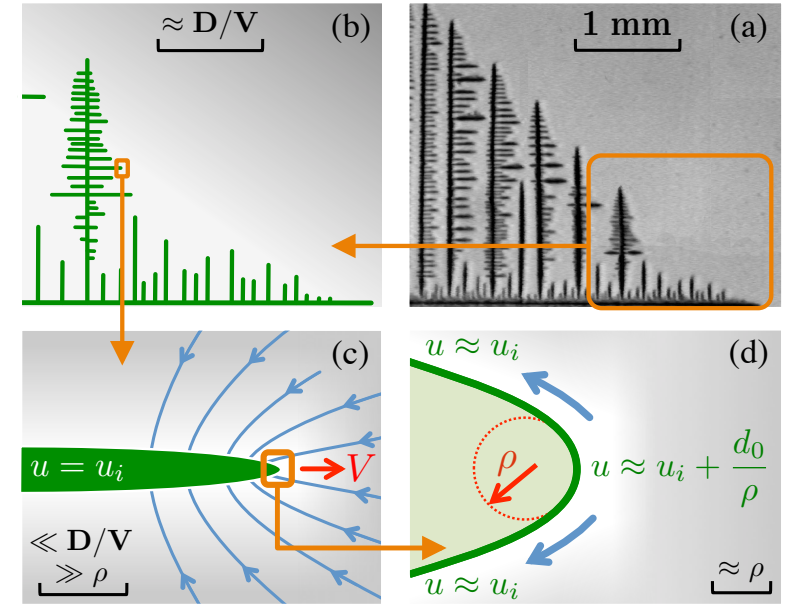

Figure 1: The Dendritic Needle Network model represents a dendritic grain, like the ammonium-bromide crystal from Ref. [38] in (a), as a hierarchical network of needle-like branches interacting through the long range diffusion field, as illustrated in (b). The instantaneous tip radius $\rho(t)$ and velocity $V(t)$ of each branch is established by combining two conditions at distinct length scales: (c) a solute conservation condition at an intermediate scale larger than the tip radius $\rho$, but smaller than the diffusion length $D / V$ (indicated with the $\gg \rho$ and $\ll D / V$ scale bar labels, respectively), and (d) a solvability condition at the scale of the tip radius $\rho$. 

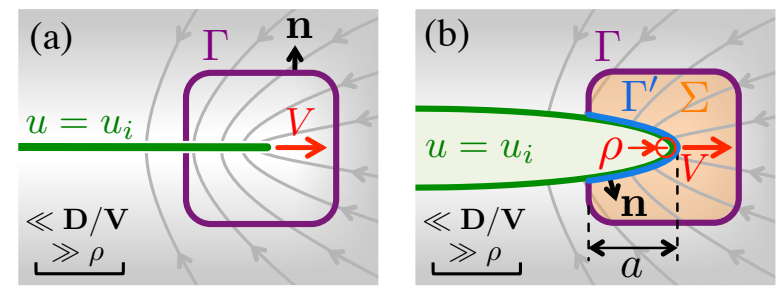

Figure 2: Schematics of a dendritic tip at an intermediate scale between the tip radius $\rho$ and the much larger diffusion length $D / V$ (indicated with the $\gg \rho$ and $\ll D / V$ scale bar labels, respectively) for (a) a sharp and (b) a parabolic needle crystal. 


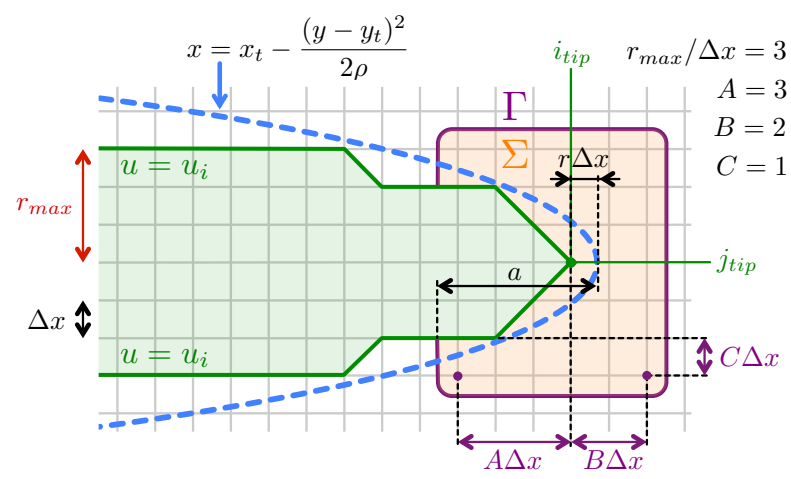

Figure 3: Illustration of a needle tip region. The integration contour (surface in 3D) $\Gamma$ and its inner surface (volume in 3D) $\Sigma$ spans $A$ grid points behind the tip location $\left(i_{t i p}, j_{t i p}\right), B$ grid points ahead of the tip, and is $C$ grid points wider than the needle at its intersection with $\Gamma$ - here illustrated for $A=3, B=2$, and $C=1$. The needle width (radius in 3D) is bounded to a maximum $r_{\max }$ - here equal to $3 \Delta x$. 


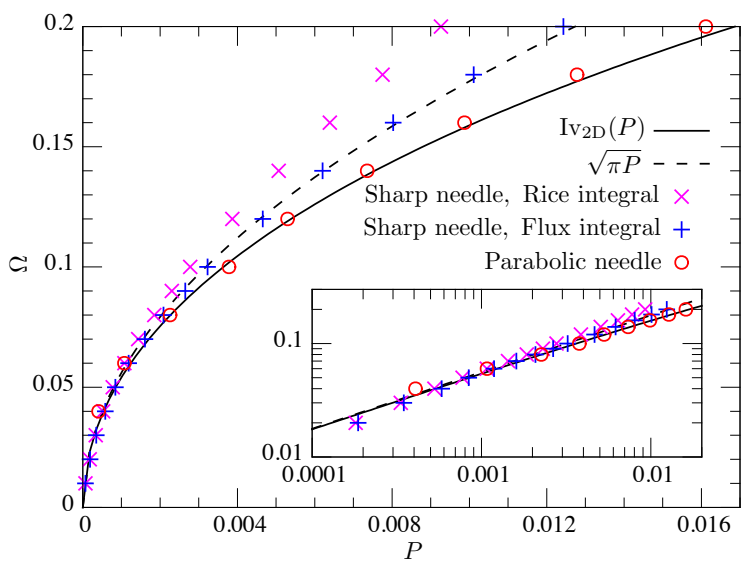

Figure 4: Steady-state Péclet number for different solute supersaturations $\Omega$ in two dimensions, compared to the exact Ivantsov solution, and its sharp-needle approximation $\Omega \approx \sqrt{\pi P}$, for three different DNN model formulations for the estimation of the flux intensity factor $\mathcal{F}:(\times)$ using the Rice integral, Eq. (16), as in Ref. [36]; (+) using the flux integral, Eq. (25), for a sharp needle; and (o) using a thick parabolic needle tip with Eqs. (25) and (29). 


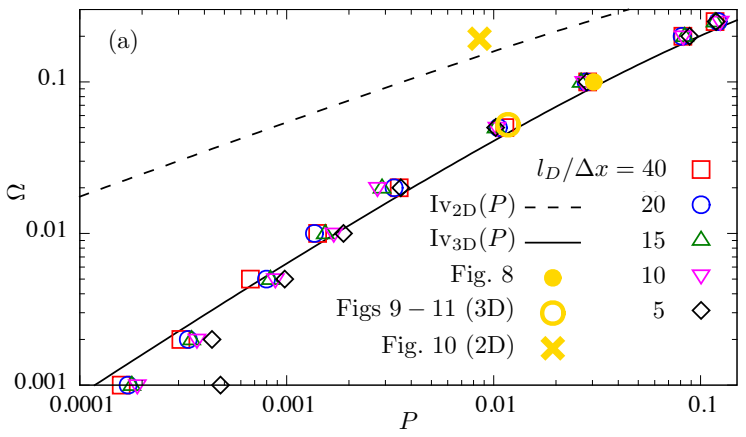

(b) $l_{D} / \Delta x=40, \Omega=0.05$

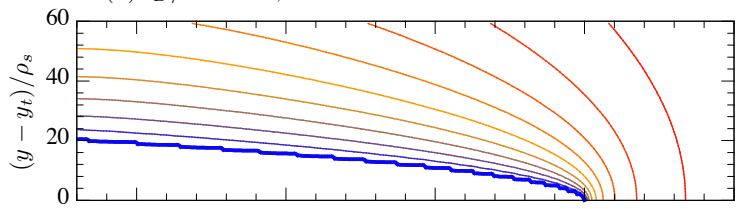

(c) $l_{D} / \Delta x=15$

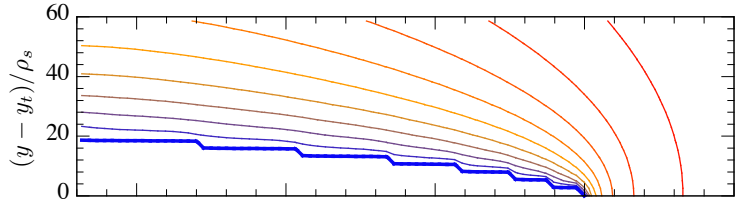

(d) $l_{D} / \Delta x=10$

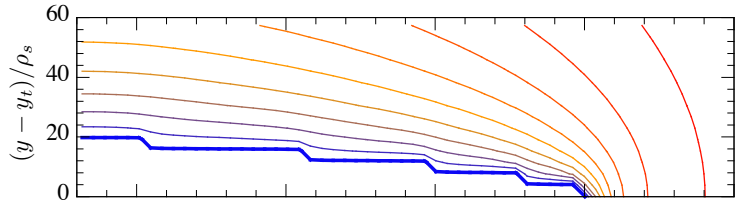

(e) $l_{D} / \Delta x=5$

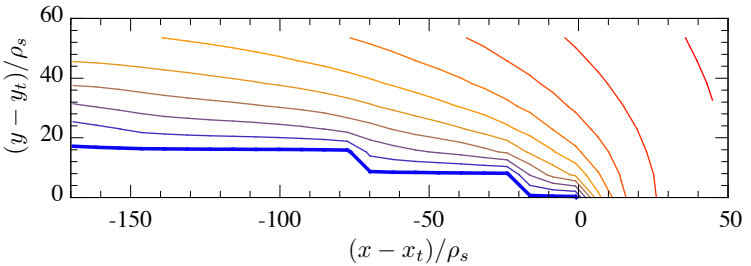

Figure 5: Steady-state supersaturation $\Omega$ versus Péclet number $P$ from DNN simulations in three dimensions with $r_{m a x}=\infty$ for $l_{D} / \Delta x=40,20,15,10$, and 5 (a). For $\Omega=0.05$, panels (b) through (e) show isovalues of the composition field $u$ in the $(z=0)$ plane with steps of 0.005 and the interface position, i.e. $u=0$, in thicker blue line, for $l_{D} / \Delta x=40$ (b), 15 (c), 10 (d), and 5 (e). Yellow symbols in (a) show the steady-state for a simulation with sidebranches (Fig. 8) and directional solidification simulations (Figs 9-11). 


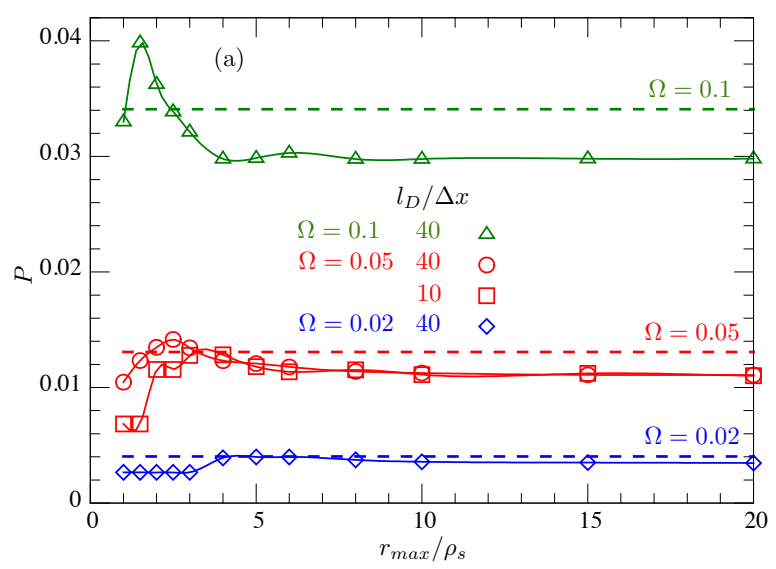

(b) $r_{\max } / \rho_{s}=20, \Omega=0.05$

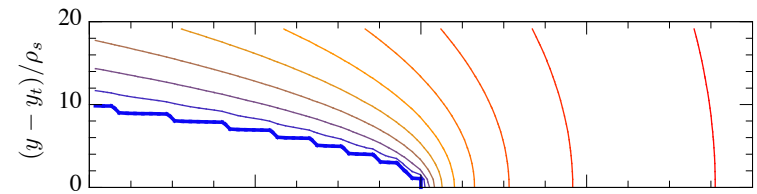

(c) $r_{\max } / \rho_{s}=8$

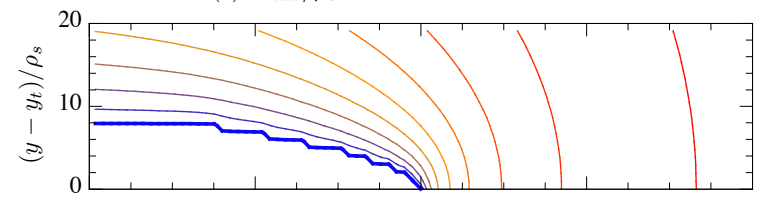

(d) $r_{\max } / \rho_{s}=5$

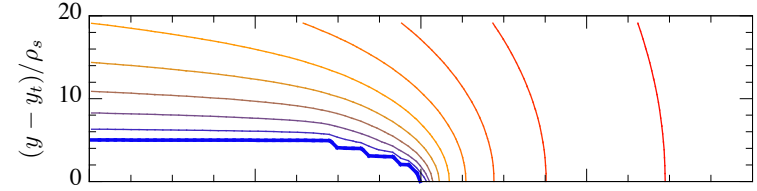

(e) $r_{\max } / \rho_{s}=3$

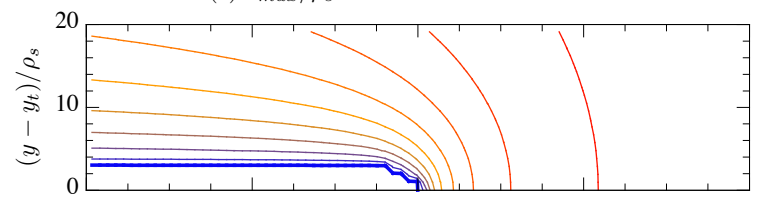

(f) $r_{\max } / \rho_{s}=1$

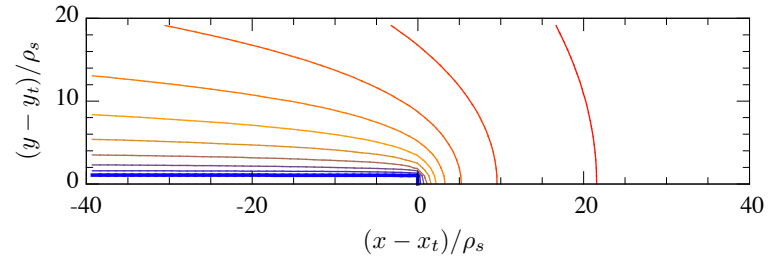

Figure 6: Predicted steady-state Péclet number $P$ as a function of the needle thickness bounding radius $r_{m a x}$ (a), and corresponding composition profiles in the $(z=0)$ plane, with contour steps of 0.005, for $\Omega=0.05, l_{D} / \Delta x=40$ and $r_{m a x} / \rho_{s}=$ 20 (b), 8 (c), 5 (d), 3 (e), and 1 (f) 

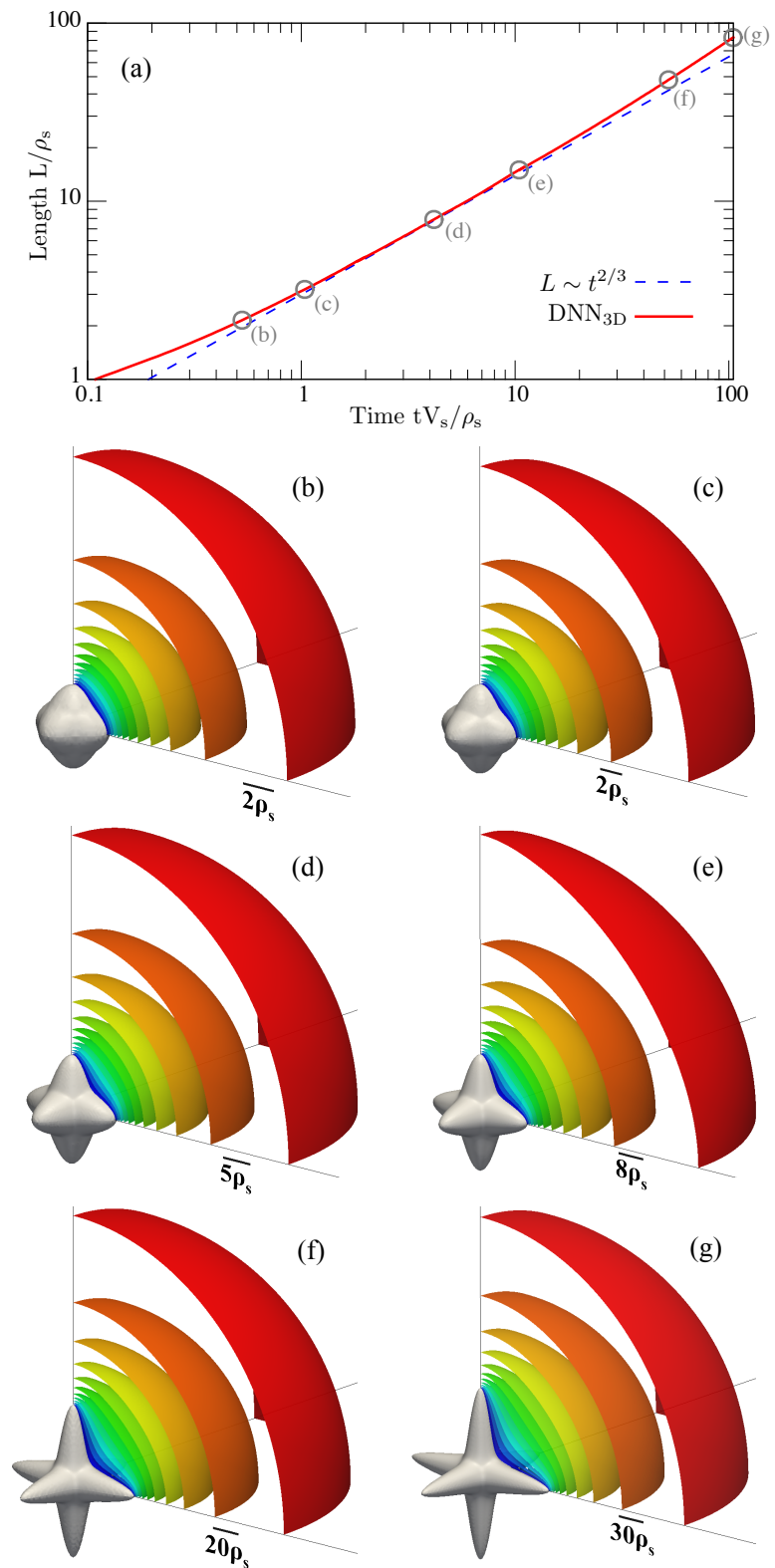

Figure 7: Early growth of a six-branch equiaxed crystal predicted by $3 \mathrm{D}$ DNN simulations for $\Omega \approx 0.00634$, i.e. $P \approx 0.00125$. In panel (a) the evolution of the branches length $L$ is compared in a log-log plot to a line of slope $2 / 3$, corresponding to the analytical law $L \sim t^{2 / 3}$ [49]. The plot is built by continuity from simulations with $\Delta x=0.05 \rho_{s}$ (for $\left.t V_{s} / \rho_{s} \leq 2\right), \Delta x=0.1 \rho_{s}$ (for $2 \leq t V_{s} / \rho_{s} \leq 10$ ), and $\Delta x=0.5 \rho_{s}$ (for $t V_{s} / \rho_{s} \geq 10$ ). Panels (b) through (g) show snapshots of the isovalues of $u$ with steps of 0.0005 , at times marked with circles in (a). 


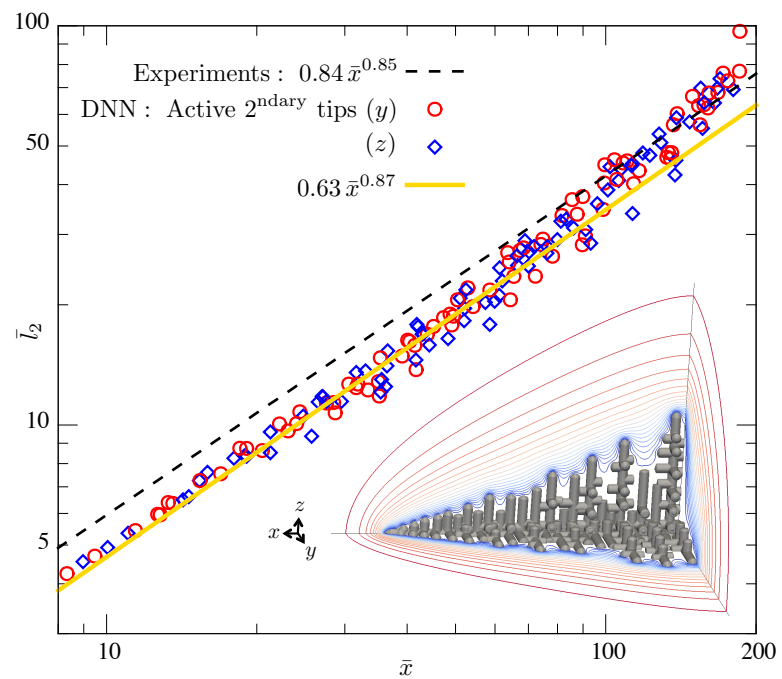

Figure 8: Sidebranches growth competition and resulting dendrite envelope for $\Omega=0.1$. Symbols mark the location of the active secondary sidebranches in the $y$ (red circles) and $z$ (blue diamonds) directions for $t V_{s} / \rho_{s}=1000$ to 2500 (shown with time steps of $250 \rho_{s} / V_{s}$ ). The power law fit of these locations (fitted with time steps of $50 \rho_{s} / V_{s}$ ) yields $\bar{l}_{2}=0.63 \bar{x}^{0.87}$ (solid line), as compared to measurements by Melendez and Beckermann [39] (dashed line). The inset shows the dendrite at $t V_{s} / \rho_{s}=1700$ with contour lines for $u=0.005$ to 0.095 with steps of 0.005 along the $(x=0),(y=0)$, and $(z=0)$ planes. 
(a)
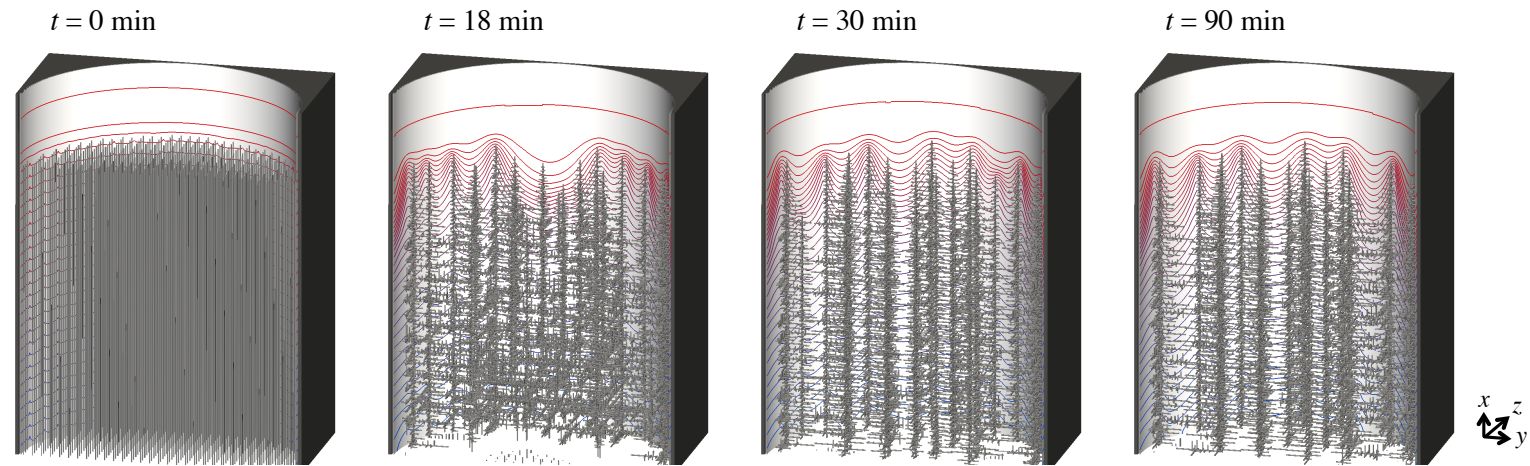

(b)
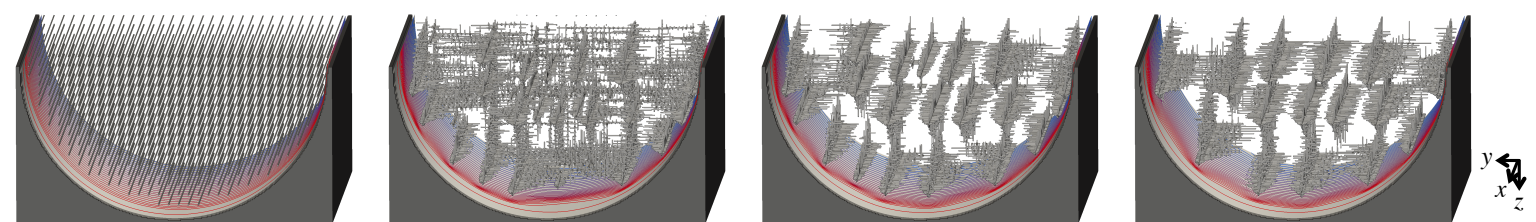

(c)
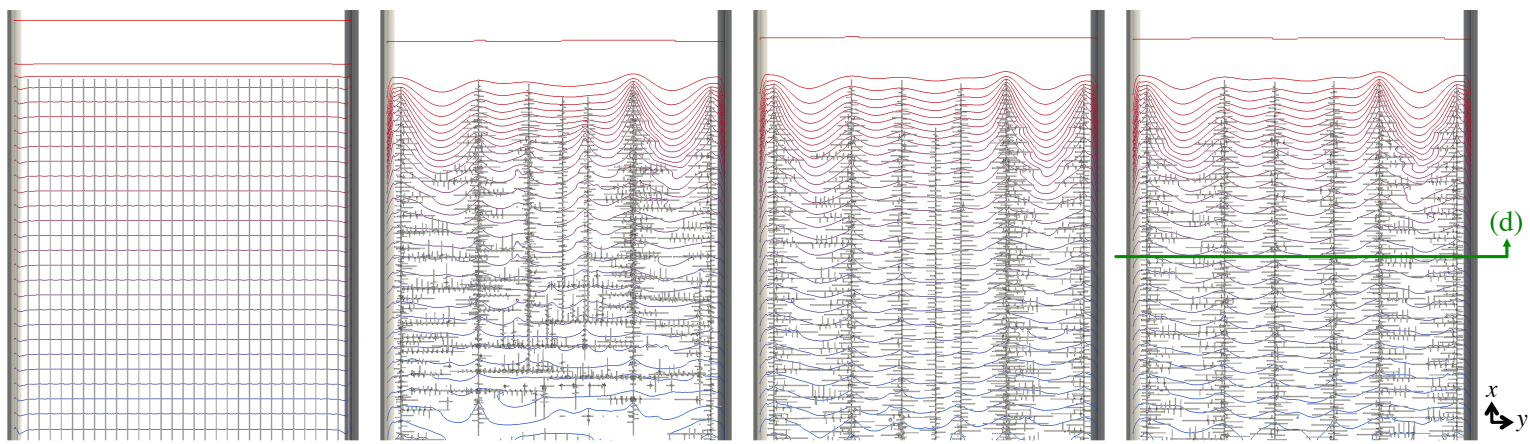

(d)
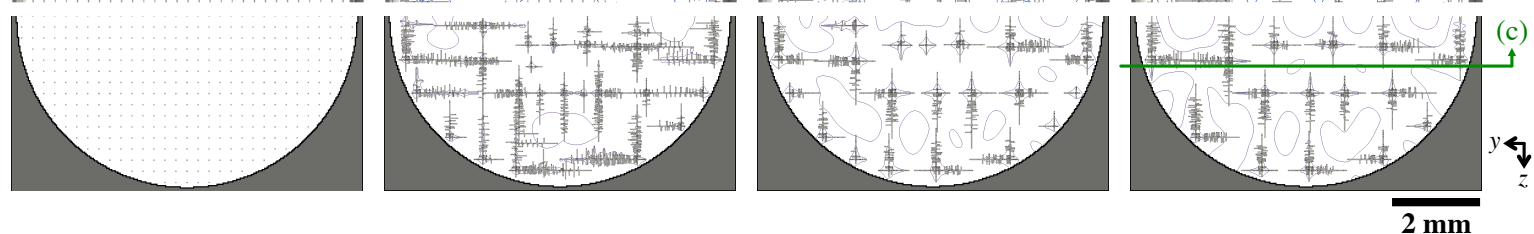

Figure 9: Snapshots of the dendrite array evolution during directional solidification of an Al-7wt.\% Si alloy at $V=10 \mu \mathrm{m} / \mathrm{s}$ and $G=9 \mathrm{~K} / \mathrm{cm}$ in a cylinder of diameter $7.8 \mathrm{~mm}$. Contour lines in (a) and (b) show isovalues of the $u$ field along the side of the cylindrical mold, with steps of 0.01. The needle network is shown in (c) for $z \leq 1121 \mu \mathrm{m}$ with isovalues of the $u$ field along the $(z=1121 \mu \mathrm{m})$ plane, and in (d) for $x \geq 4205 \mu \mathrm{m}$ with contour lines on the $(z=4205 \mu \mathrm{m})$ plane. [Both planes are drawn on the rightmost images in (c) and (d)] 


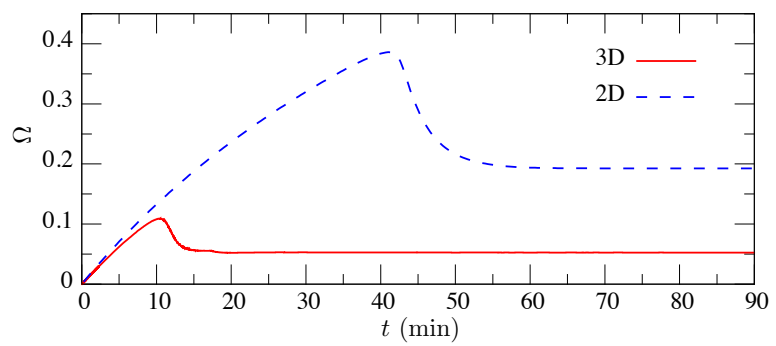

Figure 10: Transient evolution of dendritic front supersaturation for a 2D simulation from Ref. [36] (dashed line) and the present 3D simulation in a cylinder (solid). The slight difference in initial slope is due to the 2D simulation using $G=10 \mathrm{~K} / \mathrm{cm}$ (while here $G=9 \mathrm{~K} / \mathrm{cm}$ ). 
(a)
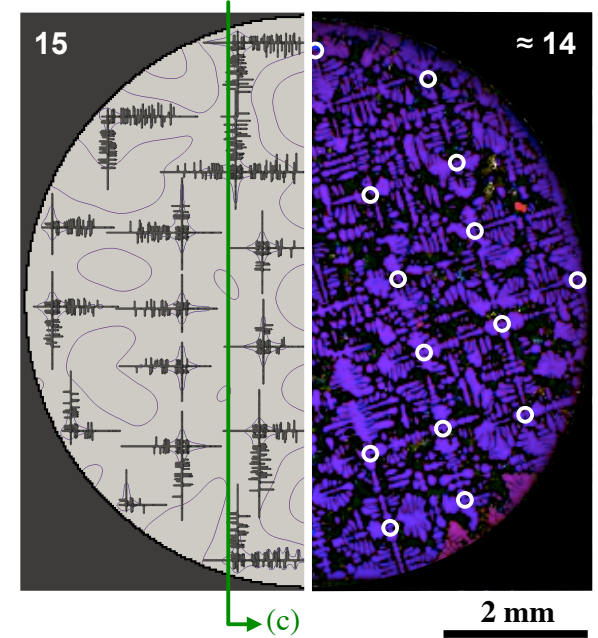

(b)

(c)

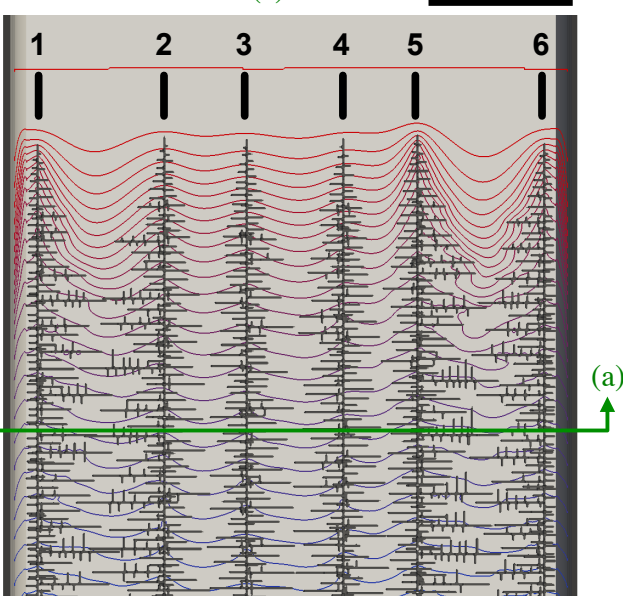

(d)

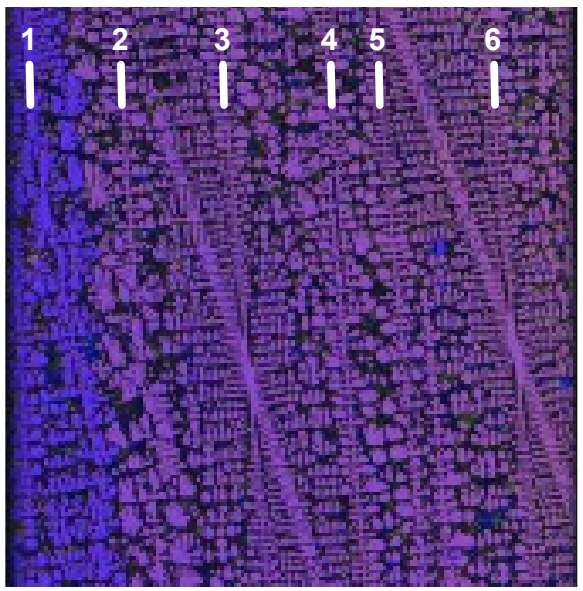

Figure 11: Microstructure cross-sections (a,b) and longitudinal sections (c,d) from a microgravity directional experiment of an Al-7wt.\%Si alloy at $V=10 \mu \mathrm{m} / \mathrm{s}$ and $G=9 \mathrm{~K} / \mathrm{cm}$ in the CETSOL project (b,d), adapted from Ref [46], and from a 3D DNN simulation $(\mathrm{a}, \mathrm{c})$. Each panel shows a count of primary dendrites. 


\section{List of Tables}

1 Parameters for Al-7wt\%Si alloy simulations. . . . . . . . . . . . . . . . . . . . . . 40 


\section{Tables}

Table 1: Parameters for Al-7wt\%Si alloy simulations.

\begin{tabular}{|c|c|c|c|}
\hline Symbol & Value & Unit & Ref. \\
\hline$c_{\infty}$ & 7 & wt\%Si & {$[46,47]$} \\
\hline$m$ & -6.5 & $\mathrm{~K} / \mathrm{wt} \% \mathrm{Si}$ & [59] \\
\hline$k$ & 0.13 & & [59] \\
\hline$\Gamma_{s l}$ & $1.9610^{-7}$ & $\mathrm{Km}$ & [59] \\
\hline$D$ & $310^{-9}$ & $\mathrm{~m}^{2} / \mathrm{s}$ & {$[3]$} \\
\hline$\sigma$ & 0.058 & & \\
\hline
\end{tabular}


